\title{
Convex Optimization-Based Compartmental Pharmacokinetic Analysis for Prostate Tumor Characterization Using DCE-MRI
}

\author{
ArulMurugan Ambikapathi, Member, IEEE, Tsung-Han Chan, Member, IEEE, Chia-Hsiang Lin, Fei-Shih Yang, \\ Chong-Yung Chi, Senior Member, IEEE, and Yue Wang
}

\begin{abstract}
Dynamic contrast enhanced magnetic resonance imaging (DCE-MRI) is a powerful imaging modality to study the pharmacokinetics in a suspected cancer / tumor tissue. The pharmacokinetic (PK) analysis of prostate cancer includes the estimation of time activity curves (TACs) and thereby the corresponding kinetic parameters (KPs), and plays a pivotal role in diagnosis and prognosis of prostate cancer. In this work, we endeavor to develop a blind source separation algorithm, namely convex optimization based KPs estimation (COKE) algorithm for PK analysis based on compartmental modeling of DCE-MRI data, for effective prostate tumor detection and its quantification. The COKE algorithm first identifies the best three representative pixels in the DCE-MRI data, corresponding to the plasma, fast flow, and slow flow TACs, respectively. The estimation accuracy of the flux rate constants (FRCs) of the fast flow and slow flow TACs directly affects the estimation accuracy of the KPs that provide the cancer and normal tissue distribution maps in the prostate region. The COKE algorithm wisely exploits the matrix structure (Toeplitz, lower triangular, and exponential decay) of the original non-convex FRCs estimation problem, and reformulates it into two convex optimization problems, that can reliably estimate the FRCs. After estimation of the FRCs, the KPs can be effectively estimated by solving a pixel-wise constrained curve-fitting (convex) problem. Simulation results demonstrate the efficacy of the proposed COKE algorithm. The COKE algorithm is also evaluated with DCE-MRI data of four different patients with prostate cancer and the obtained results are consistent with clinical observations.
\end{abstract}

Index Terms - Cancer diagnosis, Compartmental model, Convex Optimization, DCE-MRI, Kinetic parameters, Prostate cancer, Pharmacokinetic analysis, Time activity curve, Tumor characterization

This work has been partly presented in the 9th IEEE International Symposium on Biomedical Imaging (ISBI), Barcelona, Spain, May 2-5, 2012.

ArulMurugan Ambikapathi is the corresponding author. Address: Utechzone Co. Ltd., New Taipei City, Taiwan 23552, R.O.C. E-mail: aareul@ieee.org, Tel: +886-2-8226-2088X262, Fax: +886-2-82269822.

Tsung-Han Chan is with MediaTek Inc., Hsinchu, Taiwan 30078. E-mail: thchan@ieee.org, Tel: +886-919-533527.

Chia-Hsiang Lin is with Institute of Communications Engineering, National Tsing Hua University, Hsinchu, Taiwan 30013, R.O.C. E-mail: chiahsiang.steven.lin@gmail.com, Tel: +886-3-5715131X34033, Fax: +886-35751787.

Fei-Shih Yang is with Department of Radiology, Mackay Memorial Hospital, Taipei, Taiwan. E-mail: feishih-yang@umail.hinet.net, Tel: +886-225433535X2927, Fax: +886-2-25239437.

Chong-Yung Chi is with Institute of Communications Engineering \& Department of Electrical Engineering, National Tsing Hua University, Hsinchu, Taiwan 30013, R.O.C. E-mail: cychi@ee.nthu.edu.tw, Tel: +886-3-5731156, Fax: +886-3-5751787

Yue Wang is with Bradley Department of Electrical and Computer Engineering, Virginia Polytechnic Institute and State University, Arlington, VA 22203, USA. E-mail: yuewang@ vt.edu, Tel: +571-858-3150, Fax: +571-8583000 .

Copyright (c) 2014 IEEE. For permission to use this material for non-

\section{INTRODUCTION}

Prostate cancer is the most common cancer in elderly men and the number of patients with prostate cancer is considerably increasing worldwide, and so is its mortality rate [1]-[3]. Early detected prostate cancers can be more easily treated with standard therapy and the death rate can be significantly reduced [4]. Unfortunately, many patients are found to have tumors that have already spread over other surrounding tissues, by the time of their initial diagnosis of prostate cancer. Therefore, early detection of prostate tumor plays a critical role in the management of prostate cancer therapy.

Digital rectal examination (DRE) is the most common and conventional method to identify prostate cancer by elevated prostate-specific antigen (PSA) levels. The major limitation of DRE is that it is unable to detect non-palpable tumors or tumors localized in the central and transition zones of the prostate gland [5], [6]. Magnetic resonance (MR) imaging is a standard technique used for the evaluation of prostate cancer. However, it also suffers from some important limitations, like, it is very difficult to identify the cancer in central and transition zones of the prostate gland, since the tumors are visualized as weak signals with superimposed intensities on MR images [7], [8]. Dynamic contrast enhanced magnetic resonance imaging (DCE-MRI) provides a noninvasive in vivo method to evaluate tumor vasculature architectures based on contrast accumulation and washout [9], [10]. In DCE-MRI, a bolus of low molecular weight paramagnetic contrast agent (CA) is used, usually gadolinium (Gd-DTPA) [11]. The CA is transiently bounded with blood plasma and rapidly diffuses into extravascular extracellular space (EES) through capillary bed, where the relaxation processes of surrounding protons are catalyzed, and the relaxation time is shortened [12] [15]. By their inherent nature, T1-weighted DCE-MRI data are favored in the quantification of perfusion as they produce the strong signal intensity changes in the abnormal regions, when compared to the T2-weighted DCE-MRI data that have longer acquisition time. In T1-weighted DCE-MRI, instead of acquiring only one contrast enhanced image, a series of images is acquired with regular interval (approximately, every 15 to 30 seconds one image will be captured), when the CA flows in and out the concerned region of interest. Micro-vascular growth will be high in the cancer region; therefore, in the cancer region more CA passes between the vascular system and the EES tissue. Repeated MRI scans continue until the -personal purposes, contact pubs-permissions@ieee.org. 
body metabolism filters out most of the CA from the blood plasma through the kidneys (a time period usually between 6-12 minutes), and hence T1-weighted DCE-MRI produces a time series of images of the region of interest (in this work, the prostate region), and is the one considered in this work.

A signal intensity versus time curve is obtained for each pixel vector (pixel, for convenience) in the image cube and it varies in accordance with the accumulation and metabolism of CA, within the corresponding area of the prostate region [9]. While DCE-MRI can potentially depict the intra-tumor heterogeneity of vascular permeability, the quantitative application of DCE-MRI has been hindered by its inability to reliably dissect vascular compartments with distinct pharmacokinetics. For a cancerous region, the pharmacokinetic (PK) analysis of the corresponding T1-weighted DCE-MRI data is to estimate the tissue specific time activity curves (TACs) corresponding to the plasma, fast flow (cancer), and slow flow (normal) regions, and the associated kinetic parameter (KP) maps of the tissues (especially for the cancer and normal regions), for effective tumor detection. However, the prime difficulty in the analysis of the obtained T1-weighted DCE-MRI data arises due to the limited spatial resolution of the imaging modality and the partial volume effect (PVE) in the observed images [10]. Such a problem is formally referred to as the tissue heterogeneity problem, by virtue of which the observed image intensity at each pixel of DCE-MRI data set, is a weighted composition of time activities of more than one distinct tissue, irrespective of the spatial resolution of the imaging device. This inevitable PVE in DCE-MRI data hinders the quantitative PK analysis of the DCE-MRI data. To investigate this issue of PVE, many model-based approaches and algorithms have been reported for PK analysis of DCE-MRI data. They include the classical compartmental modeling (CM) [16], cluster component analysis (CCA) [17], convex analysis of mixtures with CM (CAM-CM) [18], iterative maximum likelihood CM (IML-CM) [19], and the iterative quadratic maximum likelihood (IQML) estimation [20]. In addition, modern methods such as in [21], [22] have also been suitably modified and applied to pharmacokinetic analysis. However, their major limitations include the unrealistic assumption on the compartmental model that the tissue kinetics are statistically independent [21], intractable computational complexity, and sensitivity to initialization of the unknown parameters (local optimality issues) [22].

The pharmacokinetic analysis problem has a lot in common with blind source separation (BSS), which is a signal processing methodology to extract the true sources (in PK analysis, they are the TACs and the KP maps) from the mixed observations (T1-weighted DCE-MR images), devoid of (or with very limited) prior knowledge about the sources and how those sources are mixed in the observations. In this work, for the pharmacokinetic analysis of prostate cancer using T1-weighted DCE-MRI data, we propose an effective BSS algorithm, namely convex optimization based KPs estimation (COKE) algorithm, to estimate the tissues' TACs and the KPs associated with each slice of the DCE-MRI data, for cancer detection. As in [16]-[20], this work is constructed under the premise that the prostate cancer is confirmed to be present in the patient (through biopsy tests or other clinical means). The proposed COKE algorithm intends to estimate the parameters associated with the prostate tumor through PK analysis of the DCE-MRI data of the patient taken over different positions (slices), so as to analyze the seriousness level (cancer stage) and distribution of the prostate cancer over the given region of interest.

Precisely, the proposed COKE algorithm first identifies the pure pixels (representative pixels) corresponding to the TACs, with provable theoretical guarantee. This idea is motivated by our previous work in hyperspectral image analysis (for spectral signature identification of disparate minerals in hyperspectral images for remote sensing applications) [23]. Since the sum-to-one assumption 11 in hyperspectral image analysis [23], [24] is not intrinsically satisfied in DCE-MRI data, we first normalize the DCE-MRI data and then successively estimate the pure pixels corresponding to the TACs. Once the pure pixels corresponding to the TACs are obtained, the COKE algorithm then aims to estimate the flux rate constants (FRCs). In existing methods, including the state-of-the-art CAM-CM [18] algorithm and the recently proposed TAC estimation by projection (TACE-Pro) algorithm [25], the FRCs are estimated by attempting to solve a non-convex optimization problem, thereby rendering the estimated FRCs not very reliable (due to local optimality issue) for KP estimation (cancer and normal tissue distributions). Moreover, in existing methods, the scaling ambiguities and some of the physical constraints are ignored [18], [25], which may result in unreliable estimation of the FRCs. The idea in COKE algorithm is to exploit the matrix structure (Toeplitz, lower triangular, and exponential decay) of the non-convex curve fitting problem, which results in solving two convex optimization problems to effectively estimate the FRCs, and thereby the TACs. Finally, the estimation of the KPs using the obtained TACs can be formulated as a pixelby-pixel convex constrained least-squares problem. All the convex optimization problems in the COKE algorithm can be effectively solved by available convex optimization solvers such as SeDuMi [26] and CVX [27], and due to the inherent nature of convex optimization problems, the obtained respective solutions are guaranteed to be globally optimal solutions. The simulation and experimental results are presented to demonstrate the efficacy of proposed COKE algorithm.

The ensuing sections are organized as follows. The tissue compartmental model is first presented in Section [II The transformation of the tissue compartmental model into a latent variable model, and the associated general and physical assumptions are also presented in this section. The COKE algorithm for pharmacokinetic analysis of DCE-MRI data is presented in Section III, where the idea of perspective projection and successive estimation of the pure pixel indices are presented in detail, followed by the estimation of the tissue specific FRCs and the estimation of the associated KPs. In Section IV] the proposed COKE algorithm is evaluated with synthetically generated data, under different noisy scenarios

\footnotetext{
${ }^{1}$ The sum-to-one assumption is a common assumption in some of the BSS applications, wherein the combining coefficients (also referred to as sources) in the linear mixing model sum to unity, for all observations. For instance, in hyperspectral image analysis, these coefficients are called abundances, and due to their physical constraints, they naturally sum to one.
} 


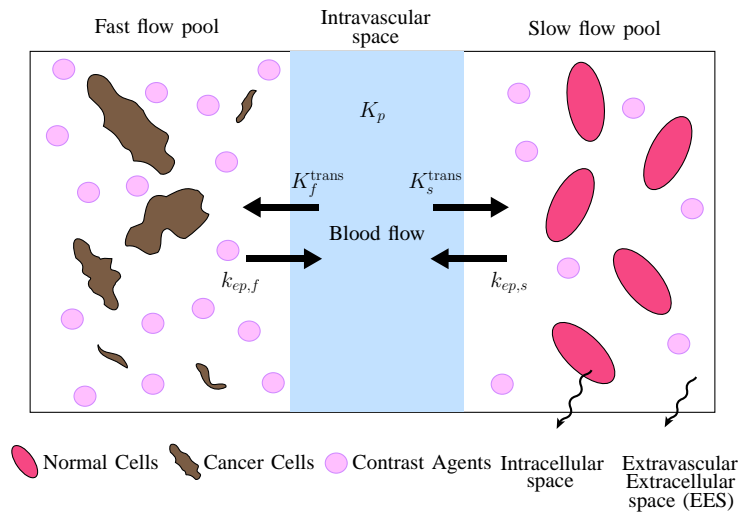

Fig. 1. Schematic diagram of three-tissue compartmental model.

and for different stages of cancer (early-stage, moderate, and advanced). The COKE algorithm is then applied to real DCEMRI data of four patients, and the results and discussions are presented in Section V Finally, Section VI provides some conclusions and future directions.

The following notations are employed in the remainder of the paper. $\mathbb{R}^{M}$ and $\mathbb{R}_{+}^{M}$ represent the set of real and non-negative real $M \times 1$ vectors, respectively. $\mathbf{1}_{N}$ and $\mathbf{I}_{N}$ represent the $N \times 1$ all-one vector, and the $N \times N$ identity matrix, respectively. $\otimes$ represents convolution operation and $\|\cdot\|_{p}$ represents the $p$-norm. $\exp (\cdot)$ stands for exponential function and $\ln (x)$ denotes natural logarithm of $x . A_{i j}$ denotes the $(i, j)$ th element of the matrix $\mathbf{A}$, and $\boldsymbol{q}_{i}(\mathbf{R})$ denotes the orthonormal eigenvector associated with the $i$ th principal eigenvalue of the positive semidefinite matrix $\mathbf{R} . \mathbf{A}^{T}$ and $\mathbf{a}^{T}$ represent the transpose of a matrix $\mathbf{A}$ and a vector $\mathbf{a}$, respectively. $\mathbf{P}_{b}^{\perp}$ is the projection matrix for the subspace orthogonal to vector $\mathbf{b}$.

\section{Signal Model and Problem Statement}

In this work, the proposed PK analysis of prostate tumor lesions using DCE-MRI time-series images is based on the well-known three-tissue compartmental model [17], [18], [29], which is a generalization of the two-tissue compartmental model proposed by Tofts et al., for the analysis of T1weighted DCE-MRI data [16], [28]. The generalized Toft's compartmental model basically consists of three tissue pools, namely fast flow pools (cancerous regions), slow flow pools (normal tissue regions), and the vascular plasma (blood), as depicted in Figure 1.

As mentioned in Figure 1, the important parameters involved in the compartmental model includes the unidirectional transfer constant $\left(K^{\text {trans }}\right)$, the flux rate constant $\left(k_{\mathrm{ep}}\right)$, plasma fractional volume $\left(K_{p}\right)$, and the extravascular extracellular space (EES). The dynamic tracer concentrations of the T1weighted DCE-MR images are governed by the following set of first-order differential equations involving the above parameters [16], [28]:

$$
\begin{aligned}
& \frac{d C_{f}(t)}{d t}+k_{\mathrm{ep}, f} C_{f}(t)=K_{f}^{\mathrm{trans}} C_{p}(t), \\
& \frac{d C_{s}(t)}{d t}+k_{\mathrm{ep}, s} C_{s}(t)=K_{s}^{\mathrm{trans}} C_{p}(t), \\
& C_{\mathrm{ms}}(t)=K_{p} C_{p}(t)+C_{f}(t)+C_{s}(t),
\end{aligned}
$$

where $C_{f}(t), C_{s}(t)$, and $C_{p}(t)$ are respectively the tracer concentrations at time $t$, in the fast flow pool, slow flow pool, and in arterial (plasma) space. $C_{\mathrm{ms}}(t)$ is the total measured tracer concentration at time $t$ and is given by the T1-weighted DCE-MR image. The unidirectional transfer coefficients from plasma to fast and slow flow pools are $K_{f}^{\text {trans }}$ and $K_{s}^{\text {trans }}$ (in $\min ^{-1}$ ), respectively. The flux rate constants from fast and slow flow pools to plasma are $k_{\mathrm{ep}, f}$ and $k_{\mathrm{ep}, s}\left(\right.$ in $\min ^{-1}$ ), respectively [9], [10]. It is straightforward to solve Equations (1)-(2) for $C_{f}(t)$ and $C_{s}(t)$ [18]. Thus we have,

$$
\begin{aligned}
C_{f}(t) & =K_{f}^{\mathrm{trans}} C_{p}(t) \otimes \exp \left(-k_{\mathrm{ep}, f} t\right), \\
C_{s}(t) & =K_{s}^{\mathrm{trans}} C_{p}(t) \otimes \exp \left(-k_{\mathrm{ep}, s} t\right) .
\end{aligned}
$$

For ease of ensuing presentation, let us define the following:

$$
\begin{aligned}
& a_{p}(t) \triangleq C_{p}(t), \\
& a_{f}(t) \triangleq C_{p}(t) \otimes \exp \left(-k_{\mathrm{ep}, f} t\right), \\
& a_{s}(t) \triangleq C_{p}(t) \otimes \exp \left(-k_{\mathrm{ep}, s} t\right) .
\end{aligned}
$$

Then (3) becomes

$$
C_{\mathrm{ms}}(t)=K_{p} a_{p}(t)+K_{f}^{\mathrm{trans}} a_{f}(t)+K_{s}^{\mathrm{trans}} a_{s}(t) .
$$

Let the temporal resolution of the T1-weighted DCE-MR images be $\Delta t$, and the tracer concentration measured at time $t_{m}=(m-1) \Delta t$ in the pixel $n$ be $C_{\mathrm{ms}}\left(n, t_{m}\right)$. Then, by (9), the temporal patterns of a given tissue slice, at pixel $n$, denoted as $\left[C_{\mathrm{ms}}\left(n, t_{1}\right), \ldots, C_{\mathrm{ms}}\left(n, t_{M}\right)\right]^{T} \in \mathbb{R}^{M}$ (where $M$ is the total number of sampling time points), can be expressed as the following latent variable model [17], [18], [29]: 2

$$
\begin{aligned}
\mathbf{x}[n] & =\left[\begin{array}{c}
C_{\mathrm{ms}}\left(n, t_{1}\right) \\
C_{\mathrm{ms}}\left(n, t_{2}\right) \\
\vdots \\
C_{\mathrm{ms}}\left(n, t_{M}\right)
\end{array}\right] \\
& =\left[\begin{array}{ccc}
a_{p}\left(t_{1}\right) & a_{f}\left(t_{1}\right) & a_{s}\left(t_{1}\right) \\
a_{p}\left(t_{2}\right) & a_{f}\left(t_{2}\right) & a_{s}\left(t_{2}\right) \\
\vdots & \vdots & \vdots \\
a_{p}\left(t_{M}\right) & a_{f}\left(t_{M}\right) & a_{s}\left(t_{M}\right)
\end{array}\right]\left[\begin{array}{c}
K_{p}[n] \\
K_{f}^{\text {trans }}[n] \\
K_{s}^{\text {trans }}[n]
\end{array}\right] \\
= & {\left[\begin{array}{lll}
\mathbf{a}_{p} & \mathbf{a}_{f} & \mathbf{a}_{s}
\end{array}\right] \mathbf{K}[n] \in \mathbb{R}^{M}, \forall n=1, \ldots, L, }
\end{aligned}
$$

where $\mathbf{x}[n]$ represents the pixel vector (will be simply referred to as pixel, for convenience) composed of temporal patterns of a given tissue slice, at pixel

\footnotetext{
${ }^{2}$ Note from $[11$ that unlike the original Toft's model [16], [28] (where both the flux rate constants and the kinetic parameters are allowed to vary from pixel to pixel, and slice to slice), in the pixel-wise generalized model given by (11), the flux rates are assumed to be constant for each slice of DCE-MRI data, so that the flux rate constants and pixel-wise kinetic parameters can be effectively estimated from the associated time series data of a slice, in the presence of measurement noise and other uncertainties.
} 
$n, \mathbf{a}_{j}=\left[a_{j}\left(t_{1}\right), \ldots, a_{j}\left(t_{M}\right)\right]^{T} \in \mathbb{R}_{+}^{M}, j \in\{p, f, s\}$ are the TACs for tissue type $j \in\{p, f, s\}$, the kinetic parameter vector $\mathbf{K}[n]=\left[K_{p}[n], K_{f}^{\text {trans }}[n], K_{s}^{\text {trans }}[n]\right]^{T}$ is the vector containing the kinetic parameters in the pixel $n$, and $L$ is the total number of pixels in the region of interest (ROI) of a given tissue slice. Specifically, $\mathbf{a}_{p} \in \mathbb{R}_{+}^{M}$ is the arterial input function (AIF) which is the plasma TAC, and $\mathbf{a}_{f} \in \mathbb{R}_{+}^{M}$ and $\mathbf{a}_{s} \in \mathbb{R}_{+}^{M}$ are the TACs of fast and slow flow tissues, respectively. Furthermore, by expressing the convolution in (7) and (8) in matrix forms, $\mathbf{a}_{f}$ and $\mathbf{a}_{s}$ can be expressed as

$$
\mathbf{a}_{j}=\mathbf{D}\left(k_{\mathrm{ep}, j}\right) \mathbf{a}_{p}, j \in\{f, s\},
$$

where $\mathbf{D}(x)$ is an $M \times M$ lower triangular matrix whose $(m, n)$ th entry is

$$
D_{m, n}(x)= \begin{cases}\Delta t e^{-(m-n) x \Delta t}, & m \geq n, \\ 0, & m<n .\end{cases}
$$

As will be seen in the ensuing sections, the judicious exploitation of the above matrix structure will be one of the vital steps of the COKE algorithm. The COKE algorithm proposed in this work for PK analysis of prostate cancer using DCE-MRI data, aims to estimate the TACs (i.e., AIF, TAC of fast flow, and TAC of slow flow) and the kinetic parameters vector associated with each pixel $n$ from the observed tracer concentration vectors $\mathbf{x}[1], \ldots, \mathbf{x}[L]$, so as to generate the kinetic parameter maps (plasma map $\mathbf{K}_{p}$, fast flow map $\mathbf{K}_{f}^{\text {trans }}$, and slow flow map $\left.\mathbf{K}_{s}^{\text {trans }}\right)$, which are defined as

$$
\begin{aligned}
\mathbf{K}_{p} & =\left[K_{p}[1], \ldots, K_{p}[L]\right]^{T} \in \mathbb{R}^{L} \\
\mathbf{K}_{j}^{\text {trans }} & =\left[K_{j}^{\text {trans }}[1], \ldots, K_{j}^{\text {trans }}[L]\right]^{T} \in \mathbb{R}^{L}, j \in\{f, s\} .
\end{aligned}
$$

Some standard (non-statistical) assumptions that have been conventionally considered by the Toft's compartmental modeling are as follows [17], [25]:

(A1) The components of the kinetic parameters vectors are non-negative i.e., $\mathbf{K}[n] \in \mathbb{R}_{+}^{3}$, for all $n$.

(A2) The TACs $\mathbf{a}_{p}, \mathbf{a}_{f}$, and $\mathbf{a}_{s}$ are linearly independent.

(A3) (Physical assumptions): $\quad 0 \leq K_{p}[n] \leq 1, \quad \forall n$, $k_{\mathrm{ep}, f} \geq K_{f}^{\operatorname{trans}}[n]$ and $k_{\mathrm{ep}, s} \geq K_{s}^{\operatorname{trans}}[n], \forall n$.

(A4) (Pure pixel assumption):

- In the entire image (with the set of indices $\mathcal{I}$ ), there exists a pure artery pixel index $l_{p} \in \mathcal{I}$ such that $K_{f}^{\text {trans }}\left[l_{p}\right]=K_{s}^{\text {trans }}\left[l_{p}\right]=0$ and $K_{p}\left[l_{p}\right] \neq 0$, thereby leading to $\mathbf{x}\left[l_{p}\right]=K_{p}\left[l_{p}\right] \mathbf{a}_{p}$.

- In the prostate gland, there exists an index set $\left\{l_{f}, l_{s}\right\}$ (i.e., "pure pixel" indices) such that $\mathbf{x}\left[l_{j}\right]=$ $K_{j}^{\text {trans }}\left[l_{j}\right] \mathbf{a}_{j}$ for $j \in\{f, s\}$.

Assumptions (A1) - (A3) are the standard straightforward assumptions that hold true in DCE-MRI data analysis [17], [25]. The pure pixel assumption, (A4) stems from the fact that within the prostate gland the distributions of the fast and slow flow tissues are not fully overlapped [25]. In other words, there exists at least a pixel location in the prostate gland such that it is purely a normal (slow flow) region (or purely a cancerous (fast flow) region). This is a practical assumption because it fails only when the entire prostate is normal or it is entirely affected by cancer. Since a major artery may not lie in the region of interest, it is assumed that in the entire image there exists a pure artery pixel, possibly corresponding to one of the major arteries such as internal pudendal artery or inferior vesical artery or middle rectal artery, of the prostate region.

\section{Convex Optimization Based Kinetic Parameter Estimation (COKE) Algorithm}

In this section, we propose a BSS algorithm, namely COKE algorithm for the PK analysis of DCE-MRI data. The COKE algorithm basically does the following: (i) Identifies the pure pixels corresponding to the TACs, (ii) Estimates the fast flow and slow flow FRCs so as to estimate the TACs, and (iii) Estimates the KP maps corresponding to the tissues, which are presented in the following subsections, respectively.

\section{A. Identification of Pure Pixel Indices}

In this subsection, we demonstrate how COKE algorithm can sequentially identify the pure pixel indices corresponding to the TACs of plasma, fast flow, and slow flow regions, from the DCE-MRI data. We begin by employing a pixel-wise normalization, that makes the KPs of the normalized pixels, sum to one (for all pixels), thereby facilitating the application of the ideas developed in [23], [30], [31] to estimate the pure pixel indices. The pixel vector normalization can be represented as:

$$
\begin{aligned}
\overline{\mathbf{x}}[n] & \triangleq \frac{\mathbf{x}[n]}{\mathbf{1}_{M}^{T} \mathbf{x}[n]} \in \mathbb{R}^{M} \\
& =\bar{k}_{p}[n] \overline{\mathbf{a}}_{p}+\bar{k}_{f}[n] \overline{\mathbf{a}}_{f}+\bar{k}_{s}[n] \overline{\mathbf{a}}_{s}, n=1, \ldots, L, \quad \text { by }
\end{aligned}
$$

where $\overline{\mathbf{a}}_{j}=\mathbf{a}_{j} /\left(\mathbf{1}_{M}^{T} \mathbf{a}_{j}\right)$ for $j \in\{p, f, s\}$ denote the normalized TACs, and $\bar{k}_{p}[n]=K_{p}[n]\left(\mathbf{1}_{M}^{T} \mathbf{a}_{p}\right) /\left(\mathbf{1}_{M}^{T} \mathbf{x}[n]\right)$ and $\bar{k}_{j}[n]=K_{j}^{\text {trans }}[n]\left(\mathbf{1}_{M}^{T} \mathbf{a}_{j}\right) /\left(\mathbf{1}_{M}^{T} \mathbf{x}[n]\right)$ for $j \in\{f, s\}$ are the normalized kinetic parameters, such that

$$
\sum_{i \in\{p, f, s\}} \bar{k}_{i}[n]=1 \text {. }
$$

In other words,

$$
\overline{\mathbf{x}}[n] \in \operatorname{conv}\left\{\overline{\mathbf{a}}_{s}, \overline{\mathbf{a}}_{f}, \overline{\mathbf{a}}_{p}\right\},
$$

where $\operatorname{conv}\{\cdot\}$ denotes the convex hull of the set of vectors, and with $\boldsymbol{\theta}=\left[\theta_{s}, \theta_{f}, \theta_{p}\right]^{T}$, it is defined as [32]:

$\operatorname{conv}\left\{\overline{\mathbf{a}}_{s}, \overline{\mathbf{a}}_{f}, \overline{\mathbf{a}}_{p}\right\}=\left\{\mathbf{x}=\sum_{i \in\{s, f, p\}} \theta_{i} \overline{\mathbf{a}}_{i} \mid \boldsymbol{\theta} \in \mathbb{R}_{+}^{3}, \mathbf{1}_{3}^{T} \boldsymbol{\theta}=1\right\}$.

The normalization procedure is visually illustrated in Figure 2 It can be seen from Figure 2 that the observed data $\mathbf{x}[n]$ (represented as green dots) are normalized (using (16), so as to satisfy the sum-to-one constraint of the normalized kinetic parameters. On account of which, the normalized data $\overline{\mathbf{x}}[n]$ (represented as red dots) are now confined to lie within the $\operatorname{conv}\left\{\overline{\mathbf{a}}_{s}, \overline{\mathbf{a}}_{f}, \overline{\mathbf{a}}_{p}\right\}$ (blue triangle and its interior) with the three extreme points (pure pixels) being $\overline{\mathbf{a}}_{s}, \overline{\mathbf{a}}_{f}$, and $\overline{\mathbf{a}}_{p}$.

So far, the signal model considered in (11) (and therefore also in (17)) does not account for the noise, which is inevitable in reality. Assuming an additive white Gaussian noise (which 


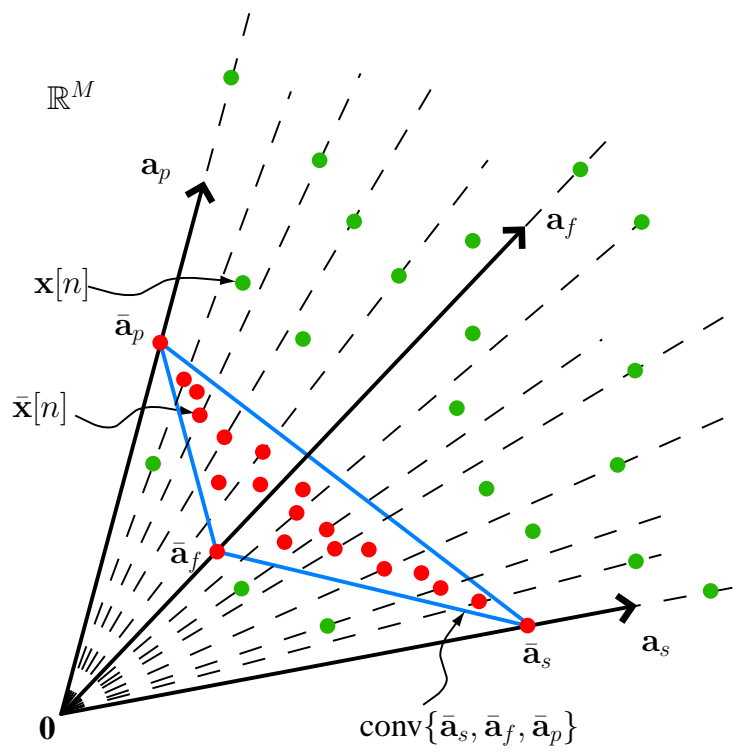

Fig. 2. Illustration of the pixel-wise normalization procedure. The pixelwise normalization projects the original data $\mathbf{x}[n]$ (shown as green dots) on the $\operatorname{conv}\left\{\overline{\mathbf{a}}_{s}, \overline{\mathbf{a}}_{f}, \overline{\mathbf{a}}_{p}\right\}$. The projected pixels $\overline{\mathbf{x}}[n]$ are represented as red dots.

is the most commonly used noise distribution in PK analysis, [18]), a dimension-reduction procedure can be employed to mitigate the noise effect and speed up the ensuing analysis. In this work, we employ the affine set fitting procedure [33], by which the dimension-reduced normalized observations $\overline{\boldsymbol{x}}[n], \forall n$ are given by

$$
\overline{\boldsymbol{x}}[n]=\mathbf{C}^{T}(\overline{\mathbf{x}}[n]-\mathbf{d}) \in \mathbb{R}^{2},
$$

where $\mathbf{C}=\left[\boldsymbol{q}_{1}\left(\mathbf{U U}^{T}\right), \boldsymbol{q}_{2}\left(\mathbf{U U}^{T}\right)\right]$, and $\mathbf{d}=\frac{1}{L} \sum_{n=1}^{L} \overline{\mathbf{x}}[n]$, in which $\mathbf{U}=[\overline{\mathbf{x}}[1]-\mathbf{d}, \ldots, \overline{\mathbf{x}}[L]-\mathbf{d}] \in \mathbb{R}^{M \times L}$. With the dimension-reduced normalized data (given by (19)), under (A1), (A2), and (A4), it has been theoretically proven in [23] that the first pure pixel index $l_{1}$ can be identified by

$$
\widehat{l}_{1} \in \arg \max _{n \in \mathcal{I}}\|\overline{\boldsymbol{x}}[n]\|_{2},
$$

where $\mathcal{I}$ is the set of pixel indices over the entire image. However, following the findings in [34] (where it has been shown that the AIF has the maximum purity among all the time series pixels in a given DCE-MRI image), it can be concluded that the so identified pure pixel corresponds to the normalized AIF i.e., $\widehat{l}_{p}=\widehat{l}_{1}$. Having identified a pure pixel index corresponding to the AIF from the entire image, we now proceed to identify the pure pixel indices corresponding to the fast flow $\left(l_{f}\right)$ and slow flow $\left(l_{s}\right)$ TACs from the prostate region of interest. Following the footsteps of the procedure 3 in [23], [25] the pure pixel indices $\left\{\widehat{l}_{2}, l_{3}\right\}=\left\{\widehat{l}_{f}, \widehat{l}_{s}\right\}$ can be

\footnotetext{
${ }^{3}$ In this work, the reliable (with theoretical guarantee for perfect identifiability), reproducible (insensitive to initializations), simplex estimation by projection (SIMPLE-Pro) algorithm introduced in [23] is used to identify the pure pixel indices. Other effective algorithms for pure pixel identification include $p$-norm based pure pixel identification algorithm (TRI-P) [23], [35], and volume maximization algorithms [30].
}

sequentially obtained as:

$$
\begin{aligned}
& \widehat{l}_{2} \in \arg \min _{n=1, \ldots, L} \overline{\boldsymbol{x}}^{T}[n] \overline{\boldsymbol{x}}\left[\widehat{l}_{p}\right], \\
& \widehat{l_{3}} \in \arg \min _{n=1, \ldots, L} \overline{\boldsymbol{x}}^{T}[n] \mathbf{d}^{\star},
\end{aligned}
$$

where $\mathbf{d}^{\star}=\mathbf{P}_{\mathbf{b}}^{\perp} \bar{x}\left[\widehat{l}_{2}\right]$, in which

$$
\mathbf{P}_{\mathbf{b}}^{\perp}=\mathbf{I}_{2}-\mathbf{b}\left(\mathbf{b}^{T} \mathbf{b}\right)^{-1} \mathbf{b}^{T},
$$

and $\mathbf{b}=\overline{\boldsymbol{x}}\left[\widehat{l}_{p}\right]-\overline{\boldsymbol{x}}\left[\widehat{l_{2}}\right]$. The above procedure has been theoretically proved to perfectly identify the pure pixel indices [23], [25].

From the estimated pure pixel indices $\left\{\widehat{l}_{2}, \widehat{l}_{3}\right\}=\left\{\widehat{l}_{f}, \widehat{l}_{s}\right\}$, the characteristics of the TACs of fast flow and slow flow pools can be used to identify $\widehat{l}_{f}$ and $\widehat{l}_{s}$. It is well known that the TAC for fast flow has a sharp peak and then a sudden decay, whereas the TAC of the slow flow has a gradual but steady increase in activity level [18], [29]. Let $\rho_{j}$ of $\mathbf{x}\left[\widehat{l}_{j}\right], j \in\{2,3\}$ be defined as

$$
\rho_{j}=\frac{\left\|\mathbf{x}\left[\widehat{l}_{j}\right]\right\|_{2}}{\left\|\mathbf{x}\left[\widehat{l}_{j}\right]\right\|_{1}}, j \in\{2,3\}
$$

Then, based on the arguments in [34], if $\rho_{2}>\rho_{3}$, then $\widehat{l}_{f}=\widehat{l}_{2}$ and $\widehat{l}_{s}=\widehat{l}_{3}$, else $\widehat{l}_{f}=\widehat{l}_{3}$ and $\widehat{l}_{s}=\widehat{l}_{2}$. Thus the pure pixel indices corresponding to the AIF, fast flow TAC, and slow flow TAC have been identified as $\widehat{l}_{p}, \widehat{l}_{f}$, and $\widehat{l}_{s}$, respectively.

\section{B. Estimation of $k_{e p, f}$ and $k_{e p, s}$}

Given the pure pixel indices $\left\{\widehat{l}_{p}, \widehat{l}_{f}, \widehat{l}_{s}\right\}$ estimated above, by (11), (12), and (A4), we have

$$
\begin{aligned}
\widehat{\mathbf{a}}_{p} & =\frac{\mathbf{x}\left[\widehat{l}_{p}\right]}{K_{p}\left[\widehat{l}_{p}\right]}, \\
\mathbf{x}\left[\widehat{l}_{f}\right] & =K_{f}^{\text {trans }}\left[\widehat{l}_{f}\right] \widehat{\mathbf{a}}_{f}=K_{f}^{\operatorname{trans}}\left[\widehat{l}_{f}\right] \mathbf{D}\left(k_{\mathrm{ep}, f}\right) \widehat{\mathbf{a}}_{p}, \\
\mathbf{x}\left[\widehat{l}_{s}\right] & =K_{s}^{\text {trans }}\left[\widehat{l}_{s}\right] \widehat{\mathbf{a}}_{s}=K_{s}^{\text {trans }}\left[\widehat{l}_{s}\right] \mathbf{D}\left(k_{\mathrm{ep}, s}\right) \widehat{\mathbf{a}}_{p} .
\end{aligned}
$$

Substituting (25) into (26) and (27) yields

$$
\mathbf{x}\left[\widehat{l}_{j}\right]=\frac{K_{j}^{\operatorname{trans}}\left[\widehat{l}_{j}\right]}{K_{p}\left[\widehat{l}_{p}\right]} \mathbf{D}\left(k_{\mathrm{ep}, j}\right) \mathbf{x}\left[\widehat{l}_{p}\right], \quad j \in\{f, s\},
$$

where $\mathbf{x}\left[\widehat{l}_{p}\right], \mathbf{x}\left[\widehat{l}_{f}\right]$, and $\mathbf{x}\left[\widehat{l}_{s}\right]$ are known. Let us emphasize that (28) holds true only when $\mathbf{x}\left[\widehat{l}_{p}\right], \mathbf{x}\left[\widehat{l}_{f}\right]$, and $\mathbf{x}\left[\widehat{l}_{s}\right]$ are noise-free measurements and the observations follow the underlying signal model in (11). In practice, the unknown kinetic parameters can be estimated by solving the following least-squares fitting problem:

$$
\min _{\substack{\left.K_{p}\left[\widehat{l}_{p}\right], K_{f}^{\text {trans }}\left[\widehat{l}_{f}\right], K_{s}^{\text {trans }} \\ k_{e p, f}, \widehat{l}_{e p, s}\right],}} \sum_{j \in\{f, s\}}\left\|\mathbf{x}\left[\widehat{l}_{j}\right]-\frac{K_{j}^{\operatorname{trans}}\left[\widehat{l}_{j}\right]}{K_{p}\left[\widehat{l}_{p}\right]} \mathbf{D}\left(k_{e p, j}\right) \mathbf{x}\left[\widehat{l}_{p}\right]\right\|_{2}^{2}
$$

subject to (s.t.) $0 \leq K_{p}\left[\widehat{l}_{p}\right] \leq 1$,

$$
0 \leq K_{j}^{\text {trans }}\left[\widehat{l}_{j}\right] \leq k_{e p, j}, j \in\{f, s\} .
$$


However, the above problem is a non-convex curve fitting problem due to the fact that the unknowns $\left\{K_{p}\left[\widehat{l}_{p}\right], K_{f}^{\operatorname{trans}}\left[\widehat{l}_{f}\right], K_{s}^{\operatorname{trans}}\left[\widehat{l}_{s}\right], k_{e p, f}, k_{e p, s}\right\} \quad$ are non-linearly combined in the objective function. Existing algorithms for kinetic parameters estimation such as CAM-CM [18], [29] and TACE-Pro [25], attempt to solve (29) using available sequential quadratic programming (SQP) solvers and hence suffer from local optimality issues in the estimation of FRCs and the ensuing KP maps. Moreover, it is also true that the methods such as CAM-CM [18], [29], simply ignore the constant $K_{p}\left[\widehat{l}_{p}\right]$ in solving (29), which may result in inaccurate estimation of FRCs and KP maps (caused due to scaling ambiguities).

Next, we will present a convex optimization based framework to estimate the FRCs and KP maps. Basically, we will exploit the structure of $\mathbf{D}\left(k_{e p, j}\right)$ in the following. Let $\widetilde{\mathbf{D}}^{j} \triangleq$ $c_{j} \mathbf{D}\left(k_{e p, j}\right), j \in\{f, s\}$, where $c_{j}=K_{j}^{\operatorname{trans}}\left[\widehat{l}_{j}\right] / K_{p}\left[\widehat{l}_{p}\right]$. Since $\widetilde{\mathbf{D}}^{j}$ is a lower-triangle Toeplitz matrix, it has the following structure:

$$
\widetilde{\mathbf{D}}^{j}=\left[\begin{array}{lcccc}
\tilde{d}_{0}^{j} & 0 & 0 & \cdots & 0 \\
\tilde{d}_{1}^{j} & \tilde{d}_{0}^{j} & 0 & \cdots & 0 \\
\tilde{d}_{2}^{j} & \tilde{d}_{1}^{j} & \tilde{d}_{0}^{j} & \cdots & 0 \\
\vdots & \vdots & \vdots & \ddots & 0 \\
\tilde{d}_{M-1}^{j} & \tilde{d}_{M-2}^{j} & \tilde{d}_{M-3}^{j} & \cdots & \tilde{d}_{0}^{j}
\end{array}\right] \in \mathbb{R}^{M \times M} .
$$

$\widetilde{\mathbf{D}}^{j}$ can be expressed as in 31) (shown at the top of next page), and based on (30), the elements of $\widetilde{\mathbf{D}}^{j}$ have the following relationship:

$$
\begin{aligned}
\widetilde{D}_{m, n}^{j} & =\widetilde{D}_{m+1, n+1}^{j} \\
& \triangleq \tilde{d}_{m-n}^{j}= \begin{cases}\tilde{d}_{r}^{j}, & 0 \leq r=m-n \leq M-1, \\
0, & \text { otherwise. }\end{cases}
\end{aligned}
$$

Then, the pharmacokinetic model fitting problem in 29) can be rewritten as the following least-squares problem, which is convex in $\widetilde{\mathbf{D}}^{j}$ :

$$
\begin{aligned}
\min _{\tilde{d}_{r}^{j}, j \in\{f, s\}, r=0, \ldots, M-1} & \sum_{j \in\{f, s\}}\left\|\mathbf{x}\left[\widehat{l}_{j}\right]-\widetilde{\mathbf{D}}^{j} \mathbf{x}\left[\widehat{l}_{p}\right]\right\|_{2}^{2} \\
\text { s.t. } & \tilde{d}_{r}^{j}=0, j \in\{f, s\}, \forall r<0, \\
& 0 \leq \tilde{d}_{r}^{j}, \tilde{d}_{r+1}^{j} \leq \tilde{d}_{r}^{j}, j \in\{f, s\}, \\
& r=0, \ldots, M-1 .
\end{aligned}
$$

Problem (32) can be solved for $\widetilde{\mathbf{D}}^{j}, j \in\{f, s\}$ by using available convex optimization solvers such as SeDuMi [26] or CVX [27]. Let the optimal solution of (32) be $\widetilde{\mathbf{D}}^{j *}$ Further, to estimate $k_{e p, f}$ and $k_{e p, s}$ from the obtained $\widetilde{\mathbf{D}}^{f *}$ and $\widetilde{\mathbf{D}}^{s *}$ respectively, we perform the following:

Step 1: Obtain the scaling factor:

$$
c_{j}=\tilde{d}_{0}^{j *} / \Delta t, j \in\{f, s\} .
$$

Step 2: Obtain the estimate of $\mathbf{D}\left(k_{e p, j}\right)$ by removing the scaling factor $c_{j}$ :

$$
\widehat{\mathbf{D}}^{j} \triangleq\left(1 / c_{j}\right) \widetilde{\mathbf{D}}^{j *}, j \in\{f, s\} .
$$

Then, from (11), (12), and (A4), the AIF and other TACs are estimated as

$$
\begin{aligned}
\mathbf{a}_{p} K_{p}\left[\widehat{l}_{p}\right] & =\mathbf{x}\left[\widehat{l}_{p}\right] \approx \widehat{\mathbf{a}}_{p}, \\
\widehat{\mathbf{a}}_{j} & =\widehat{\mathbf{D}}^{j} \widehat{\mathbf{a}}_{p}, j \in\{f, s\} .
\end{aligned}
$$

Note that the above estimated AIF (i.e., the plasma TAC) still has a scaling ambiguity, as $K_{p}\left[\widehat{l}_{p}\right]$ is still unknown. This scaling ambiguity also propagates to $\widehat{\mathbf{a}}_{f}$ and $\widehat{\mathbf{a}}_{s}$. However, this issue of scaling ambiguity will be appropriately handled while estimating the KP maps, which is discussed in the next subsection.

Since from (13), $\ln D_{m, 1}^{j}=\ln \Delta t-k_{e p, j}(m-1) \Delta t, m=$ $1, \ldots, M, j \in\{f, s\}$, from the estimated $\widehat{\mathbf{D}}^{j}$, the flux rate constants $\left(k_{e p, f}\right.$ and $\left.k_{e p, s}\right)$ can be obtained by using available convex optimization solvers [26], [27], to solve the following convex problem:

$$
\begin{aligned}
\min _{k_{e p, f}, k_{e p, s}} & \sum_{m=1}^{M} \sum_{j \in\{f, s\}}\left\|\ln \widehat{D}_{m, 1}^{j}-\ln \Delta t+k_{e p, j}(m-1) \Delta t\right\|_{2}^{2} \\
\text { s.t. } & 0 \leq k_{e p, j}, j \in\{f, s\}, \\
& k_{e p, s} \leq k_{e p, f} .
\end{aligned}
$$

Once, the AIF estimate $\left(\widehat{\mathbf{a}}_{p}\right)$, TAC estimates $\left(\widehat{\mathbf{a}}_{f}\right.$, and $\left.\widehat{\mathbf{a}}_{s}\right)$, and FRC estimates $\left(\widehat{k}_{e p, f}\right.$ and $\left.\widehat{k}_{e p, s}\right)$ are estimated, the KP maps can be estimated as discussed next.

\section{Estimation of KP Maps}

Finally, the estimation of tissue distribution maps (i.e., kinetic parameter maps), based on (11) boils down to the following convex least-squares fitting problem, for $n=1,2, \ldots, L$ :

$$
\begin{aligned}
\widehat{\mathbf{K}}[n]=\arg \min _{\mathbf{K}[n]} & \left\|\mathbf{x}[n]-\alpha\left[\widehat{\mathbf{a}}_{p}, \widehat{\mathbf{a}}_{f}, \widehat{\mathbf{a}}_{s}\right] \mathbf{K}[n]\right\|_{2}^{2} \\
\text { s.t. } \quad & 0 \leq K_{p}[n] \leq 1, \\
& K_{f}^{\text {trans }}[n] \geq 0, K_{s}^{\text {trans }}[n] \geq 0 \\
& K_{s}^{\text {trans }}[n] \leq \widehat{k}_{e p, s}, K_{f}^{\text {trans }}[n] \leq \widehat{k}_{e p, f}
\end{aligned}
$$

The scaling parameter $\alpha$ in (38) is used to alleviate the scaling ambiguity caused due to the unknown $K_{p}\left[\widehat{l}_{p}\right]$. Setting a suitable scaling factor $\alpha$ (say $\alpha=100$ ), (38) can be solved by using available convex optimization solvers like SeDuMi [26] and CVX [27]. If this scaling is not appropriately taken into account, the KP maps estimated by (38) will be an overestimation of the true KP maps, as the estimated KP vectors may reach their respective upper bounds (i.e., saturation levels) owing to the constraints of (38), thus violating the relationship among $K_{p}[n], K_{f}^{\text {trans }}[n]$, and $K_{s}^{\text {trans }}[n]$. It should be noted that the $K_{p}[n], K_{f}^{\text {trans }}[n]$, and $K_{s}^{\text {trans }}[n]$, estimated are relative values of their respective true values, however their relationships are maintained, by preventing them from reaching the saturation level. The only condition on choosing the value of $\alpha$ is that it should be larger than the inverse of $K_{p}\left[l_{p}\right]$. More importantly, such a value of $\alpha$ will not affect the distribution of the estimated KP maps, as will also be addressed in Sections IV and V] below. Also, it is worth 


$$
\widetilde{\mathbf{D}}^{j}=c_{j} \underbrace{\mathbf{D}\left(k_{e p, j}\right) \text { defined by (13) }}_{\left.\begin{array}{lcccc}
\Delta t & 0 & 0 & \cdots & 0 \\
\Delta t e^{-k_{e p, j} \Delta t} & \Delta t & 0 & \cdots & 0 \\
\Delta t e^{-k_{e p, j} 2 \Delta t} & \Delta t e^{-k_{e p, j} \Delta t} & \Delta t & \cdots & 0 \\
\vdots & \vdots & \vdots & \ddots & 0 \\
\Delta t e^{-k_{e p, j}(M-1) \Delta t} & \Delta t e^{-k_{e p, j}(M-2) \Delta t} & \Delta t e^{-k_{e p, j}(M-3) \Delta t} & \cdots & \Delta t
\end{array}\right]}
$$

mentioning that unlike in [18], the estimation of $K_{s}^{\operatorname{trans}}[n]$ and $K_{f}^{\text {trans }}[n]$ are respectively upper bounded by $\widehat{k}_{e p, s}$ and $\widehat{k}_{e p, f}$, thereby avoiding the possible over estimation of the transfer constants (and thereby the KP maps). Thus, all the kinetic parameters are estimated with low sensitivity to the over estimation (saturation) issue. It should be noted that the optimization problems (32), (37), and 38) involved in estimating the kinetic parameters are all convex optimization problems for which the solutions are guaranteed to be global optimal [32]. The pseudo-code for the entire COKE algorithm is given in Table 1 .

\section{Simulations}

In this section, we study the performance of the proposed COKE algorithm on synthetically generated DCE-MRI data. This way of performance evaluation on synthetic data is very important to study the validity of the estimates obtained by an algorithm under test, as it may be the only means to verify the effectiveness of an PK analysis algorithm, since exact ground truths are not available for real DCE-MRI data. It has been recently shown that the CAM-CM algorithm [18] has better performance when compared to several existing PK analysis algorithms. Moreover, unlike COKE and CAMCM, other algorithms such as IML-CM [19], and IQML [20], assume tissue homogeneity which is seldom true in reality. Hence in this section, the performance of the proposed COKE algorithm will be solitarily compared with the performance of CAM-CM algorithm. The two algorithms are evaluated for their accuracies in estimating the flux rate constants $\left(k_{\mathrm{ep}, f}\right.$ and $\left.k_{\mathrm{ep}, s}\right)$. The mean \pm standard deviation of the flux rate constants estimated by the algorithms under test, over 50 independent runs, for each of the different scenarios is used as the performance measure for the estimated flux rate constants.

The simulation settings for generating the synthetic data are as follows: The AIF $\mathbf{a}_{p}$ is generated based on the population average mode 4 [36], with a temporal resolution $\Delta t=4$ seconds for 7 -min period $(M=105)$. Since the fast flow and the slow flow TACs (given by (12) are characterized by $k_{\mathrm{ep}, f}$ and $k_{\mathrm{ep}, s}$, respectively, they are generated based on the values of $k_{\mathrm{ep}, f}$ and $k_{\mathrm{ep}, s}$ in each scenario. In this section, for the sake of simulating different stages of prostate cancer, there are three scenarios under consideration. Note that in the following scenarios, the range for generating the kinetic parameters are

\footnotetext{
${ }^{4}$ The population average model is a well-known model used for artificially generating the AIF. It is primarily used to synthesize the AIF (using candidate TACs in two reference tissues) for PK analysis, in cases where the AIF cannot be directly obtained from the DCE-MR images.
}

TABLE I

PSEUDOCODE FOR COKE ALGORITHM.

Given Observed DCE-MRI data $\mathbf{x}[n] \in \mathbb{R}^{M}, n=1 \ldots, L$, the temporal resolution of the T1-weighted DCE-MR images $\Delta t$, and a scaling factor $\alpha$ (say $\alpha=100$ ).

Step 1. Compute the normalized data, $\overline{\mathbf{x}}[n]$ for each $n=1 \ldots, L$ by using 16 .

Step 2. Obtain the dimension-reduced data $\overline{\boldsymbol{x}}[n]$ from the normalized data $\overline{\mathbf{x}}[n]$ by using

$$
\overline{\boldsymbol{x}}[n]=\mathbf{C}^{T}(\overline{\mathbf{x}}[n]-\mathbf{d}) \in \mathbb{R}^{2},
$$

where $\mathbf{C}$ and $\mathbf{d}$ are defined along with 19 .

Step 3. Identify the pure pixel index corresponding to the normalized AIF by

$$
\widehat{l}_{p} \in \arg \max _{n \in \mathcal{I}}\|\overline{\boldsymbol{x}}[n]\|_{2},
$$

where $\mathcal{I}$ is the set of pixel indices over the entire image.

Step 4. Identify the pure pixel indices $\widehat{l}_{2}$ and $\widehat{l}_{3}$ by

$$
\begin{aligned}
& \left.\widehat{l}_{2} \in \arg \min _{n=1, \ldots, L} \overline{\boldsymbol{x}}^{T}[n] \overline{\boldsymbol{x}} \widehat{l}_{p}\right], \\
& \widehat{l}_{3} \in \arg \min _{n=1, \ldots, L} \overline{\boldsymbol{x}}^{T}[n] \mathbf{d}^{\star},
\end{aligned}
$$

where $\mathbf{d}^{\star}=\mathbf{P}_{\mathbf{b}}^{\perp} \overline{\boldsymbol{x}}\left[\widehat{l}_{2}\right]$, in which $\mathbf{P}_{\mathbf{b}}^{\perp}$ is given by 23 .

Step 5. Compute $\rho_{2}$ and $\rho_{3}$ given by (24). Assign $\widehat{l}_{f}=\widehat{l}_{2}$ and $\widehat{l}_{s}=\widehat{l}_{3}$, if $\rho_{2}>\rho_{3}$; else, $\widehat{l}_{f}=\widehat{l}_{3}$ and $\widehat{l}_{s}=\widehat{l}_{2}$.

Step 6. Define the latent matrix $\widetilde{\mathbf{D}}^{j} \in \mathbb{R}^{M \times M}$ by specifying its elements as following, for each $j \in\{f, s\}$ :

$$
\begin{aligned}
\widetilde{D}_{m, n}^{j} & =\widetilde{D}_{m+1, n+1}^{j} \\
& \triangleq \tilde{d}_{m-n}^{j}= \begin{cases}\tilde{d}_{r}^{j}, & 0 \leq r=m-n \leq M-1, \\
0, & \text { otherwise. }\end{cases}
\end{aligned}
$$

Then solve the convex problem given by 32 and obtain the optimal $\widetilde{\mathbf{D}}^{f *}$ and $\widetilde{\mathbf{D}}^{s *}$. Compute $c_{j}=\widetilde{d}_{0}^{j *} / \Delta t$ and $\widehat{\mathbf{D}}^{j}=\left(1 / c_{j}\right) \cdot \widetilde{\mathbf{D}}^{j *}$, for each $j \in\{f, s\}$.

Step 7. Compute the TACs: $\widehat{\mathbf{a}}_{p} \approx \mathbf{x}\left(\widehat{l}_{p}\right)$ and $\widehat{\mathbf{a}}_{j}=\widehat{\mathbf{D}}^{j} \widehat{\mathbf{a}}_{p}$, for each $j \in\{f, s\}$.

Step 8. Solve the convex problem in 37 and obtain the flux rate constants $\left\{\widehat{k}_{e p, s}, \hat{k}_{e p, f}\right\}$.

Step 9. Obtain $\widehat{\mathbf{K}}[n]$ by solving the least-squares (convex) fitting problem given in 38, for each $n=1, \ldots, L$.

Output The TACs (given by Step 7), the FRCs (given by Step 8), and the KP maps $\left(\widehat{\mathbf{K}}_{f}^{\text {trans }}, \widehat{\mathbf{K}}_{s}^{\text {trans }}, \widehat{\mathbf{K}}_{p}\right.$ ) (defined by (14) and 15 , and given by Step $\mathbf{9}$ ).

chosen such that the assumptions (A1) and (A3) hold true for the generated data set. The three scenarios under consideration are:

Scenario 1: Early-stage tumor with parameters $k_{\mathrm{ep}, f}=$ 1.625 , and $\left\{K_{f}^{\text {trans }}[n]\right\}_{n=1}^{L}$ randomly generated following a uniform distribution over the interval $[0.4,0.6]$. 


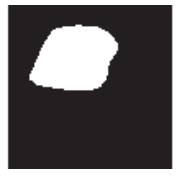

Plasma Region

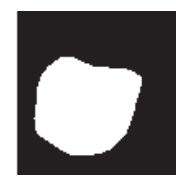

Fast Flow Region

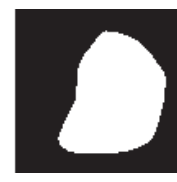

Slow Flow Region

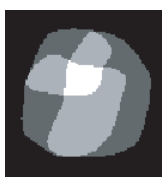

Tumor ROI
Fig. 3. The fast flow, slow flow, plasma, and the tumor ROI (comprising fast flow, slow flow, and plasma) regions, used in the simulations [18].

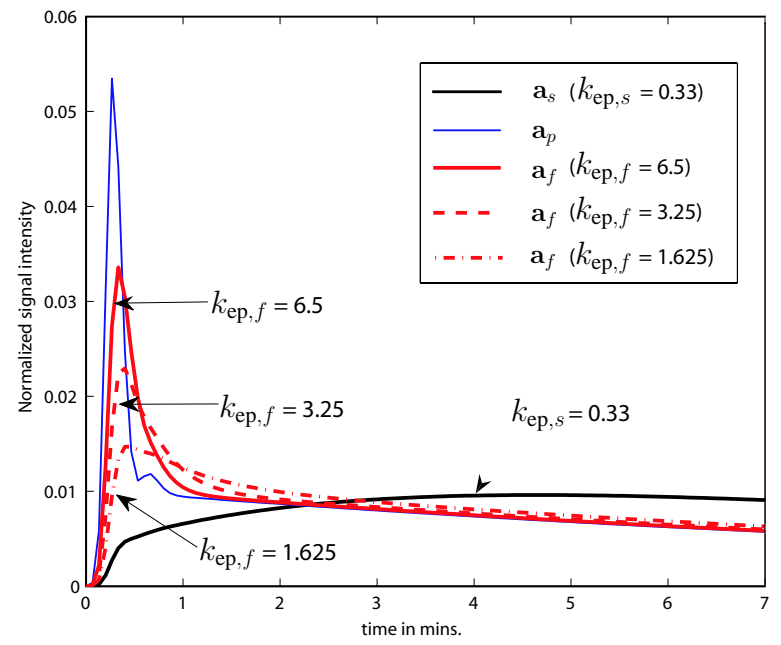

Fig. 4. Typical TAC curves for fast flow (for different $k_{\mathrm{ep}, f}$ values), slow flow and plasma (AIF).

Scenario 2: Moderate-stage tumor with parameters $k_{\mathrm{ep}, f}=$ 3.25 , and $\left\{K_{f}^{\text {trans }}[n]\right\}_{n=1}^{L}$ randomly generated following a uniform distribution over the interval $[0.8,1.2]$.

Scenario 3: Advanced-stage tumor with parameters $k_{\mathrm{ep}, f}=$ 6.5 , and $\left\{K_{f}^{\text {trans }}[n]\right\}_{n=1}^{L}$ randomly generated following a uniform distribution over the interval $[1.7,2.3]$.

In all the scenarios $k_{\mathrm{ep}, s}=0.33$, is set to be a constant. The fast flow, slow flow, and the plasma regions are chosen as in [18] and are shown in Figure 3 The tissue regions for fast flow, slow flow, and plasma are not fully overlapped and hence meet the pure pixel assumption (A4). The parameters $\left\{K_{s}^{\text {trans }}[n]\right\}_{n=1}^{L}$ and $\left\{K_{p}[n]\right\}_{n=1}^{L}$ are uniformly generated over the interval $[0.08,0.12]$ and $[0.04,0.06]$, respectively. Finally, the synthetic data set $\mathbf{x}[n]$, for all $n=1, \ldots, L$, is obtained following the linear mixing model in (11). For each data set generated using the regions used in [18], the number of pixels within the ROI is around 5000 , i.e., $L \approx 5000$. The typical TAC curves are shown in Figure 4 for reference, and will be handy in comparing the results of real data experiments that will be presented in Section $\mathrm{V}$

Then, the synthetically generated pixel vectors $\mathbf{x}[n]$ are added with Gaussian white noise with zero mean and covariance matrix $\sigma^{2} \mathbf{I}_{M}$ based on a given signal-to-noise ratio (SNR) which is defined as

$$
\mathrm{SNR} \triangleq \frac{\sum_{n=1}^{L}\|\mathbf{x}[n]\|_{2}^{2}}{\sigma^{2} M L} .
$$

The SNR levels considered are $20 \mathrm{~dB}, 25 \mathrm{~dB}, 30 \mathrm{~dB}, 35 \mathrm{~dB}$, $40 \mathrm{~dB}$, and $\infty$ (noise-free case).

The mean \pm standard deviation of the estimated FRCs $\left(\widehat{k}_{\mathrm{ep}, f}\right.$ and $\widehat{k}_{\text {ep }, s}$ ) obtained by the algorithms under test are tabulated in Table III It can be seen from Table II] that in all the 3 scenarios, especially for lower SNR cases (high noise cases), the COKE algorithm outperforms the state-of-the-art CAM$\mathrm{CM}$ algorithm (employed with the best possible parameter set). Also, as the SNR increases, the mean values of the estimates (obtained by COKE and CAM-CM) get closer to the true values of the respective flux rate constants, and the standard deviation approaches to zero. However, the CAM$\mathrm{CM}$ algorithm fails under the noise-free scenario, as the algorithm is built under the premise of noise presence in the observed DCE-MRI data. Thus, Table II demonstrates the validity and superior efficacy of the proposed COKE algorithm over the benchmark and state-of-the-art CAM-CM algorithm. The performance of the proposed COKE algorithm using real data will be demonstrated in the ensuing section.

\section{EXPERIMENTAL RESUlts}

Having demonstrated the efficacy of the COKE algorithm using synthetic data sets, in this section, we apply the COKE algorithm to four real DCE-MRI data obtained from four different patients suffering from prostate cancer. The DCEMRI data sets were provided by Mackay Memorial Hospital, Taipei, Taiwan and were acquired by using Philips Achieva with a 3-Tesla magnetic field strength. The acquired threedimensional data set with $4 \mathrm{~mm}$ slice thickness, $0.45 \mathrm{~mm}$ pixel spacing, $10^{\circ}$ field of view, and in-plane matrix size $256 \times 256$, was taken every 30 seconds for a total of 10 minutes after the injection of Gadolinium DTPA, for each patient. The four male patients referenced as Patients A, B, C, and D are aged $72,75,68$, and 87 , respectively. For all the four data sets, the suspected abnormal areas were marked in a corresponding T2-weighted image, by a radiologist, and those corresponding areas in the T1-weighted DCE-MR images are considered for marking the ROI for the real data experiments. It should be mentioned that except for the AIF selection procedure (as explained in Section V.A below), the application of COKE algorithm is confined to this marked ROI in each slice of a patient, and so are the ensuing results and discussions. The biopsy tests have been conducted for all the four patients and the test results for all the patients in the three regions (anterior, middle, and posterior) of the prostate cancer, are shown in Table III. The values indicated in Table IIII are the percentage of cancerous tissues in the biopsy samples taken at the respective locations. Specifically, the biopsy examination has been conducted in 12 locations, for each patient; six locations in the peripheral prostate region and six locations in the central prostate regions. The biopsy observations are made through the anus, and in the following discussions, the anterior and posterior regions correspond to smaller and larger slice numbers, respectively. It should be noted that the biopsy results are based on samples obtained in some specific locations (as it is not possible to extract biopsy samples in all the different slices with the slice thickness set to $4 \mathrm{~mm}$, which 
TABLE II

MEAN \pm STANDARD DEVIATION OF THE ESTIMATED FLUX RATE CONSTANTS $\left(\widehat{k}_{\mathrm{ep}, f}, \widehat{k}_{\mathrm{ep}, s}\right)$ OBTAINED BY COKE AND CAM-CM OVER 50 INDEPENDENT RUNS, FOR DIFFERENT RANDOM TISSUE MAPS AND DIFFERENT SNRS. $k_{\mathrm{ep}, s}=0.33$ IN ALL CASES (NA STANDS FOR "NOT APPLICABLE").

\begin{tabular}{|c|c|c|c|c|c|c|c|}
\hline \multirow{2}{*}{$\begin{array}{c}\text { SNR } \\
(\mathrm{dB})\end{array}$} & \multirow{2}{*}{$\widehat{k}_{\mathrm{ep}}$} & \multicolumn{2}{|c|}{$\begin{array}{c}\text { Early-stage tumor } \\
k_{\mathrm{ep},}=1.625\end{array}$} & \multicolumn{2}{c|}{$\begin{array}{c}\text { Moderate tumor } \\
k_{\mathrm{ep}, f}=3.25\end{array}$} & \multicolumn{2}{c|}{$\begin{array}{c}\text { Advanced tumor } \\
k_{\mathrm{ep}, f}=6.5\end{array}$} \\
\cline { 3 - 8 } & & $\mathrm{COKE}$ & CAM-CM & COKE & CAM-CM & COKE & CAM-CM \\
\hline \multirow{2}{*}{20} & $\widehat{k}_{\mathrm{ep}, f}$ & $1.83 \pm 0.17$ & $2.10 \pm 0.05$ & $3.45 \pm 0.31$ & $4.14 \pm 0.08$ & $6.33 \pm 1.03$ & $8.50 \pm 0.66$ \\
\cline { 2 - 8 } & $\widehat{k}_{\mathrm{ep}, s}$ & $0.39 \pm 0.02$ & $0.45 \pm 0.01$ & $0.40 \pm 0.02$ & $0.45 \pm 0.00$ & $0.41 \pm 0.02$ & $0.47 \pm 0.01$ \\
\hline \multirow{2}{*}{25} & $\widehat{k}_{\mathrm{ep}, f}$ & $1.77 \pm 0.13$ & $1.91 \pm 0.03$ & $3.47 \pm 0.18$ & $3.79 \pm 0.06$ & $6.84 \pm 0.71$ & $7.57 \pm 0.13$ \\
\cline { 2 - 8 } & $k_{\mathrm{ep}, s}$ & $0.36 \pm 0.01$ & $0.40 \pm 0.00$ & $0.36 \pm 0.01$ & $0.40 \pm 0.00$ & $0.37 \pm 0.01$ & $0.41 \pm 0.00$ \\
\hline \multirow{2}{*}{30} & $\widehat{k}_{\mathrm{ep}, f}$ & $1.74 \pm 0.08$ & $1.79 \pm 0.01$ & $3.48 \pm 0.17$ & $3.57 \pm 0.03$ & $6.78 \pm 0.45$ & $7.13 \pm 0.07$ \\
\cline { 2 - 8 } & $\widehat{k}_{\mathrm{ep}, s}$ & $0.35 \pm 0.01$ & $0.37 \pm 0.00$ & $0.35 \pm 0.01$ & $0.37 \pm 0.00$ & $0.35 \pm 0.01$ & $0.38 \pm 0.00$ \\
\hline \multirow{2}{*}{35} & $\widehat{k}_{\mathrm{ep}, f}$ & $1.69 \pm 0.06$ & $1.72 \pm 0.00$ & $3.40 \pm 0.14$ & $3.44 \pm 0.00$ & $6.71 \pm 0.28$ & $6.87 \pm 0.00$ \\
\cline { 2 - 8 } & $\widehat{k}_{\mathrm{ep}, s}$ & $0.34 \pm 0.00$ & $0.35 \pm 0.00$ & $0.34 \pm 0.00$ & $0.35 \pm 0.00$ & $0.34 \pm 0.00$ & $0.36 \pm 0.00$ \\
\hline \multirow{2}{*}{40} & $\widehat{k}_{\mathrm{ep}, f}$ & $1.68 \pm 0.05$ & $1.69 \pm 0.00$ & $3.39 \pm 0.11$ & $3.36 \pm 0.00$ & $6.77 \pm 0.25$ & $6.71 \pm 0.00$ \\
\cline { 2 - 8 } & $k_{\mathrm{ep}, s}$ & $0.34 \pm 0.00$ & $0.34 \pm 0.00$ & $0.34 \pm 0.00$ & $0.34 \pm 0.00$ & $0.34 \pm 0.00$ & $0.35 \pm 0.00$ \\
\hline \multirow{2}{*}{$\infty$} & $\widehat{k}_{\mathrm{ep}, f}$ & $1.63 \pm 0.00$ & NA & $3.25 \pm 0.00$ & NA & $6.50 \pm 0.00$ & NA \\
\cline { 2 - 8 } & $\widehat{k}_{\mathrm{ep}, s}$ & $0.33 \pm 0.00$ & NA & $0.33 \pm 0.00$ & NA & $0.33 \pm 0.00$ & NA \\
\hline
\end{tabular}

is both painful and tedious), and hence could only serve as a partial reference for comparison with the respective KP maps obtained for the different patients. Table III will be handy in comparison and validation of the estimated KP maps in Section $\mathrm{V}-\mathrm{C}$

\section{A. Estimation of Time Activity Curves}

The proposed COKE algorithm, summarized in Table I, is used to estimate the TACs (AIF, fast flow TAC, and slow flow TAC), FRCs, and the KP maps (with $\alpha=100$ ), of interes 5 . As discussed in Section $\Pi$ here, the "purest pixel" corresponding to the AIF is chosen from the entire scanned region (not just confined to the ROI), for each tissue slice of a patient, based on (20). Furthermore, among the AIFs (purest pixels) chosen for the different tissue slices of a patient, the purest pixel that has the best visual match with the standard AIF (shown in Fig. 4) is used universally for the estimation of fast flow and slow flow TACs, of each tissue slice of that particular patient. The reason behind choosing the AIF globally for all slices of a patient is due to the practical fact that the AIF may not be explicitly available / captured in each of the DCE-MRI slices of a patient. For ease of visualization, for each Patient, the TACs estimated by COKE algorithm for 5 affected tissue slices are shown in Fig. 5 , where the slice number represents the position of the MRI scanner that scans the prostate region from bottom to top. Also for the purpose of comparison and validation, the purest pixels in the data i.e., $\mathbf{x}\left[l_{f}\right]$ and $\mathbf{x}\left[l_{s}\right]$, corresponding to fast flow TAC and slow flow TAC, respectively are shown (dotted lines) along with the estimated TACs (solid lines) for various slices. It can be readily observed from Fig. 5 that for most cases, the estimated TACs have shapes similar to those of the respective purest pixels in the data. Moreover, while the patterns of the TACs obtained by COKE for Patients A and $\mathrm{B}$ are close to those of the ideal TAC curves (shown in

\footnotetext{
${ }^{5}$ For other values of $\alpha=500,1000$, the KP maps remain the same and only the values in the color bars are different.

${ }^{6}$ High-resolution images of Figures 5 to 9 , and additional simulations using real AIFs estimated from the patients, are available at http://www.ee.nthu.edu.tw/cychi/publications-e.html
}
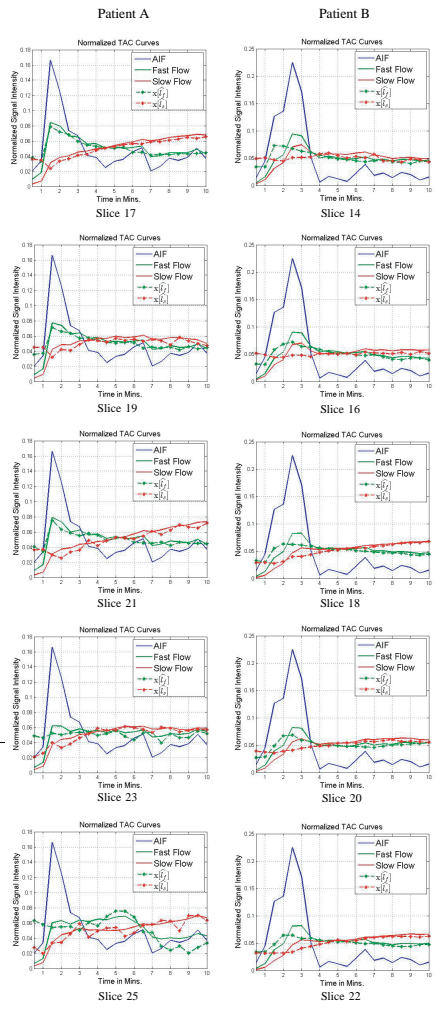

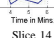
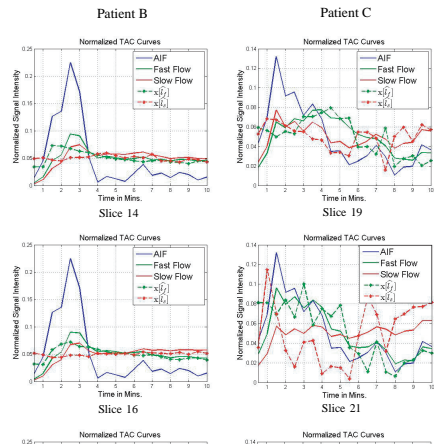

Sirnominis.
Slice 19

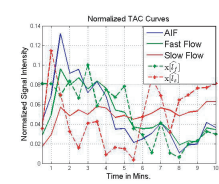

Timb himing
Slice 21

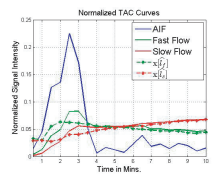

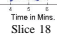
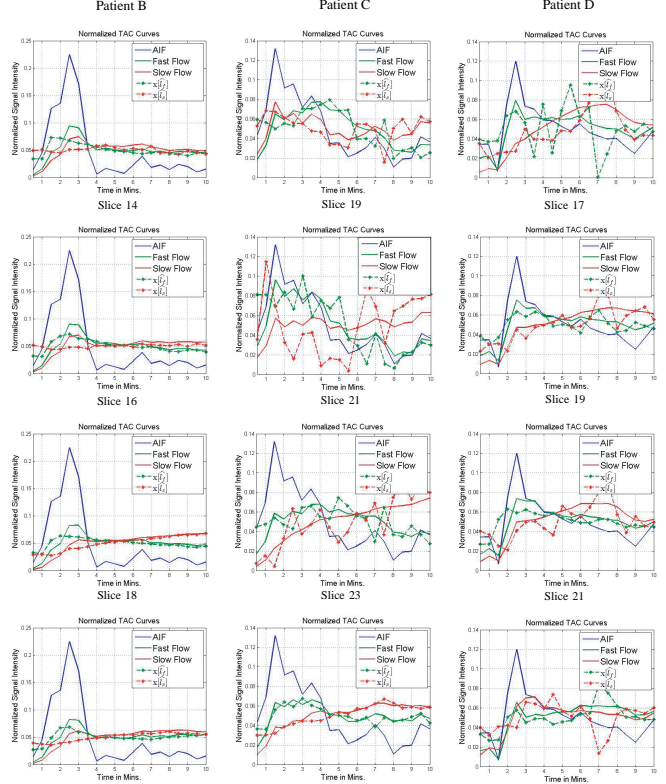

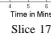

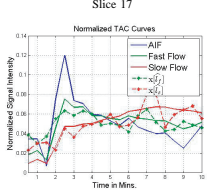

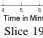

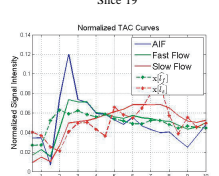

Trmening
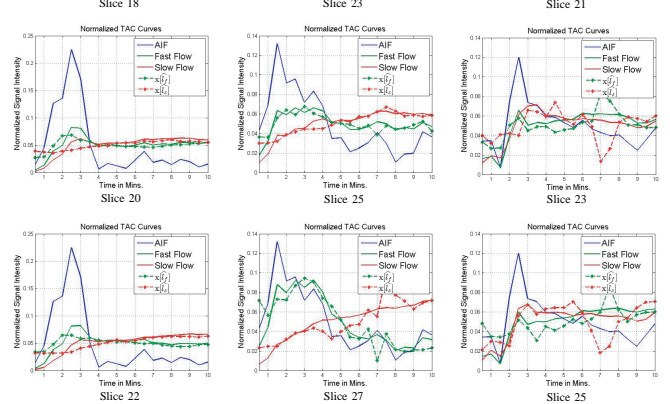

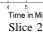

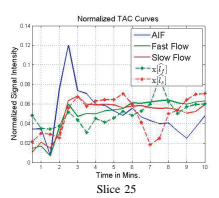

Fig. 5. The fast flow TAC (green solid line) and the slow flow TAC (red solid line) estimated by COKE algorithm, for different tissue slices of each Patient. The associated fast flow pure pixel $\mathbf{x}\left[l_{f}\right]$ (green dotted line) and the slow flow pure pixel $\mathbf{x}\left[l_{s}\right]$ (red dotted line) in the data, are also shown.

Fig. 4), those deformations in TACs obtained for Patients $\mathrm{C}$ and $\mathrm{D}$ could be attributed to the inevitable noise present in the data, or due to the unavailability of a perfect pure pixel corresponding to the AIF, fast flow TAC, slow flow TAC, or all the above.

\section{B. Estimation of Flux Rate Constants}

The $\widehat{k}_{\mathrm{ep}, f}$ and $\widehat{k}_{\mathrm{ep}, s}$ values obtained by the COKE algorithm for the respective tissue slices for the four patients are also 
TABLE III

THE BIOPSY RESULTS (INFERRED PERCENTAGE OF TUMOR DISTRIBUTION) FOR PATIENTS A, B, C, AND D FOR DIFFERENT POSITIONS. IN THIS TABLE, PERIPHERY AND CENTER LOCATIONS CORRESPOND TO THE PERIPHERAL AND CENTRAL PROSTATE REGIONS, RESPECTIVELY.

\begin{tabular}{|c|c|c|c|c|c|c|c|}
\hline Patients & Locations & Anterior-Left & Anterior-Right & Middle-Left & Middle-Right & Posterior-Left & Posterior-Right \\
\hline \multirow{2}{*}{ Patient A } & Periphery & $80 \%$ & $95 \%$ & $90 \%$ & $90 \%$ & $90 \%$ & $90 \%$ \\
\hline & Center & $90 \%$ & $50 \%$ & $45 \%$ & $95 \%$ & $85 \%$ & $95 \%$ \\
\hline \multirow{2}{*}{ Patient B } & Periphery & $0 \%$ & $10 \%$ & $0 \%$ & $5 \%$ & $0 \%$ & $0 \%$ \\
\hline & Center & $0 \%$ & $0 \%$ & $0 \%$ & $0 \%$ & $0 \%$ & $0 \%$ \\
\hline \multirow{2}{*}{ Patient $\mathrm{C}$} & Periphery & $0 \%$ & $35 \%$ & $0 \%$ & $40 \%$ & $0 \%$ & $20 \%$ \\
\hline & Center & $0 \%$ & $30 \%$ & $0 \%$ & $30 \%$ & $0 \%$ & $65 \%$ \\
\hline \multirow{2}{*}{ Patient D } & Periphery & $0 \%$ & $95 \%$ & $0 \%$ & $95 \%$ & $0 \%$ & $95 \%$ \\
\hline & Center & $0 \%$ & $95 \%$ & $0 \%$ & $95 \%$ & $0 \%$ & $95 \%$ \\
\hline
\end{tabular}

tabulated in Table IV] Specifically, it can be observed from Tables [V] that the dynamic range of the FRCs estimated by COKE is regulated between 0 and 2. It should be emphasized that the estimated FRC values are dependent upon the imaging device and the preset imaging intensity. Nevertheless, these $\widehat{k}_{\mathrm{ep}, f}$ and $\widehat{k}_{\mathrm{ep}, s}$ values serve as a measure to identify the intensity of cancer versus normal tissue. In fact, the larger the difference between these two values, the severer is the cancer intensity, and vice versa. As per this fact, for instance, based on $\widehat{k}_{\mathrm{ep}, f}$ and $\widehat{k}_{\mathrm{ep}, s}$ estimated by COKE algorithm (from Table IV], it can be concluded that slices 17 and 21 of Patient A, slices 18 and 22 of Patient B, slices 23 and 27 of Patient C, and slices 17 and 19 of Patient D, reveal advanced stage cancers. This inference is also consistent with the Gleason score [37] (that ranges from 2 to 10) of the patients, which are 7, 6, 8, and 8, for Patients A, B, C, and D, respectively, as a higher Gleason score indicates advanced cancers [37].

\section{Estimation of Kinetic Parameter Maps}

The obtained KP maps (fast flow map $\widehat{\mathbf{K}}_{f}^{\text {trans }}$, slow flow map $\widehat{\mathbf{K}}_{s}^{\text {trans }}$, and plasma map $\widehat{\mathbf{K}}_{p}$ ), within the manually selected respective ROI, for different slices, are shown in Figs. 6 to 9, for the four patients (Patient A to Patient D), respectively. In Figs. 6 to 9, the ROIs in the prostate region of a slice are shown along with the entire obtained DCE-MR image in that slice position, to show the relative variations of the ROI with respect to different slices, as the ROIs are manually marked for each slice of a patient. The color bars in Figs. 6 to 9, that are shown adjacent to the kinetic parameter maps indicate the level of activeness of the particular tissue. More specifically, if the colors in the map of slow flow tissue are close to the maximum value (dark red) of its color bar, it indicates that the tissue is quite normal. On the other hand, if the colors in the map of fast flow tissue are close to the maximum value (dark red) of its color bar, then it indicates dominant detected cancer tissues. However, the seriousness level of the detected cancer tissues can be decided only based on the estimated FRC values (cf. Section V-B). As expected, it can be observed that the slow flow and fast flow maps basically complement each other in all the results shown in Figs. 6 to 9. It can also be observed that for each patient, the detected tumor regions (fast flow maps) in each slice varies; indicating the irregular spread of tumor tissues in and over the prostate region.
The obtained KP maps are also consistent with the biopsy results shown in Table IIII For instance, for Patient $A$ the biopsy results suggest that the tumor is present almost in all parts of the prostate, which is consistent with the obtained KP maps for Patient A (shown in Fig. 6). More importantly, the obtained KP maps could yield more information (which is the prime purpose for PK analysis) than the ones available only based on the biopsy results. To see this, let us first consider Patient B (Fig. 7). Slice 14 (anterior portion) reveals that there is cancer on the left side of the slice, which coincides with Table [III. The biopsy results in Table III claim that there are no cancers in other regions. However the other slices in Fig. 7 do indicate the irregular spread of the cancer to the other regions of the prostate, which is also clinically verified by the doctors team. Similar inference can be made for Patient C (Fig. 8) and Patient D (Fig. 9). It is worth reiterating the fact that the biopsy examination results can only serve as a reference and more information regarding the actual spread and distribution of the tumor can be obtained only through PK analysis of the data. Also, one can observe from Figs. 6 to 9 that the fast flow KP maps associated with those slices mentioned in Section $\mathrm{V}-\mathrm{B}$ above (slices 17 and 21 of Patient A, slices 18 and 22 of Patient B, slices 23 and 27 of Patient C, and slices 17 and 19 of Patient D) indeed reveal the presence of cancer tissues in the ROI.

\section{Additional Discussions}

The CAM-CM algorithm [18] has been designed for PK analysis based on compartmental modeling (irrespective of the type of cancers). However, the results (TACs, FRCs, and KP maps) yielded by applying CAM-CM to real prostate data are uninterpretable 7 . The reasons for the inapplicability of CAM-CM for prostate tumor detection could be attributed to the following: (i) The AIF estimation procedure for COKE is more practical and realistic (cf. Section $\mathrm{V}-\mathrm{A}$ ), than the AIF estimation procedure in CAM-CM. (ii) The ignorance of the scaling constant and the local optimality effects due to non-convexity of the problems involved in CAM-CM may have significant impact on the results of real data experiments. (iii) In addition, there are also quite some tuning parameters involved in CAM-CM. The default tuning parameters are based on the simulated data (where the true information about

\footnotetext{
${ }^{7}$ For reference, the PK analysis results using CAM-CM, for the four patients and the respective slices are available at http://www.ee.nthu.edu.tw/cychi/publications-e.html
} 
TABLE IV
The FRCs $\left(\widehat{k}_{\mathrm{ep}, f}, \widehat{k}_{\mathrm{ep}, s}\right)$ EStimated B y COKE FOR PATIENT A, B, C, AND D FOR DiffERENT SLICES

\begin{tabular}{|c|c|c|c|c|c|c|}
\hline \multirow{3}{*}{ Patient A } & FRCs & Slice 17 & Slice 19 & Slice 21 & Slice 23 & Slice 25 \\
\cline { 2 - 7 } & $k_{\mathrm{ep}, f}$ & 0.6495 & 0.5693 & 0.5779 & 0.3711 & 0.4802 \\
\cline { 2 - 7 } & $\mathfrak{k}_{\mathrm{ep}, s}$ & 0.1451 & 0.3012 & 0.1274 & 0.2262 & 0.1693 \\
\hline \multirow{3}{*}{ Patient B } & FRCs & Slice 14 & Slice 16 & Slice 18 & Slice 20 & Slice 22 \\
\cline { 2 - 7 } & $\widehat{k}_{\mathrm{ep}, f}$ & 0.3289 & 0.3535 & 0.2986 & 0.2648 & 0.2834 \\
\cline { 2 - 7 } & $\boldsymbol{k}_{\mathrm{ep}, s}$ & 0.2547 & 0.2067 & 0.0786 & 0.1435 & 0.0749 \\
\hline \multirow{3}{*}{ Patient C } & FRCs & Slice 19 & Slice 21 & Slice 23 & Slice 25 & Slice 27 \\
\cline { 2 - 7 } & $\boldsymbol{k}_{\mathrm{ep}, f}$ & 0.5437 & 1.1541 & 0.4831 & 0.3886 & 1.2377 \\
\cline { 2 - 7 } & $\boldsymbol{k}_{\mathrm{ep}, s}$ & 0.4163 & 0.3154 & 0.0383 & 0.2512 & 0.1089 \\
\hline \multirow{3}{*}{ Patient D } & FRCs & Slice 17 & Slice 19 & Slice 21 & Slice 23 & Slice 25 \\
\cline { 2 - 7 } & $\widehat{k}_{\mathrm{ep}, f}$ & 0.6049 & 0.6485 & 0.5470 & 0.4220 & 0.4363 \\
\cline { 2 - 7 } & $\mathfrak{k}_{\mathrm{ep}, s}$ & 0.1401 & 0.2730 & 0.2946 & 0.3896 & 0.3414 \\
\hline
\end{tabular}

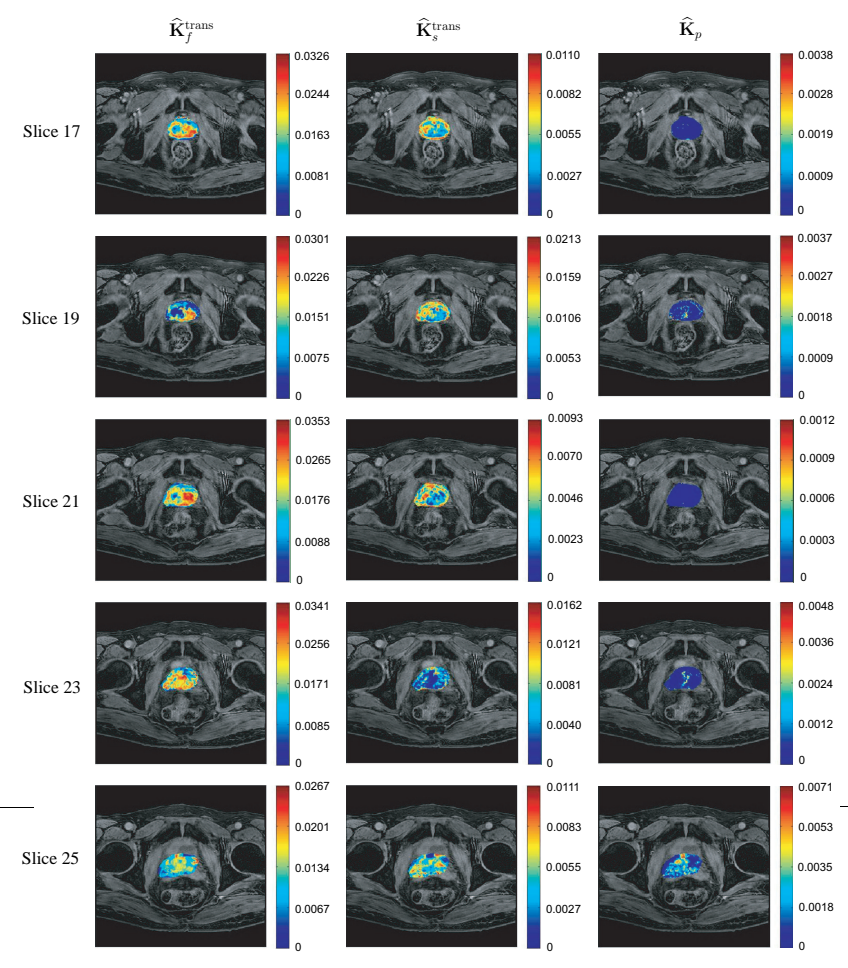

Fig. 6. The KP Maps Estimated by COKE algorithm for Patient A in different slices.

the TACs and FRCs is available), and hence for the simulated data CAM-CM exhibited performance comparable to that of COKE (cf. Table П). However, for the real data experiments on prostate cancer, the optimal tuning parameters for CAM-CM are unknown and difficult to find, and this is out of the scope of this paper. Furthermore, as reported in [18], the CAM$\mathrm{CM}$ algorithm has been validated for the breast cancer data, which are less prone to motion effects, as the DCE-MR image sequences were externally fixed. Whereas, the prostate cancer data suffers from motion effects jointly due to the motion caused through breathing and bowel movement. In addition to the above mentioned algorithmic issues, such inevitable motion effects and noise artifacts could also have resulted in poor performance of the CAM-CM algorithm when applied to the prostate cancer data.

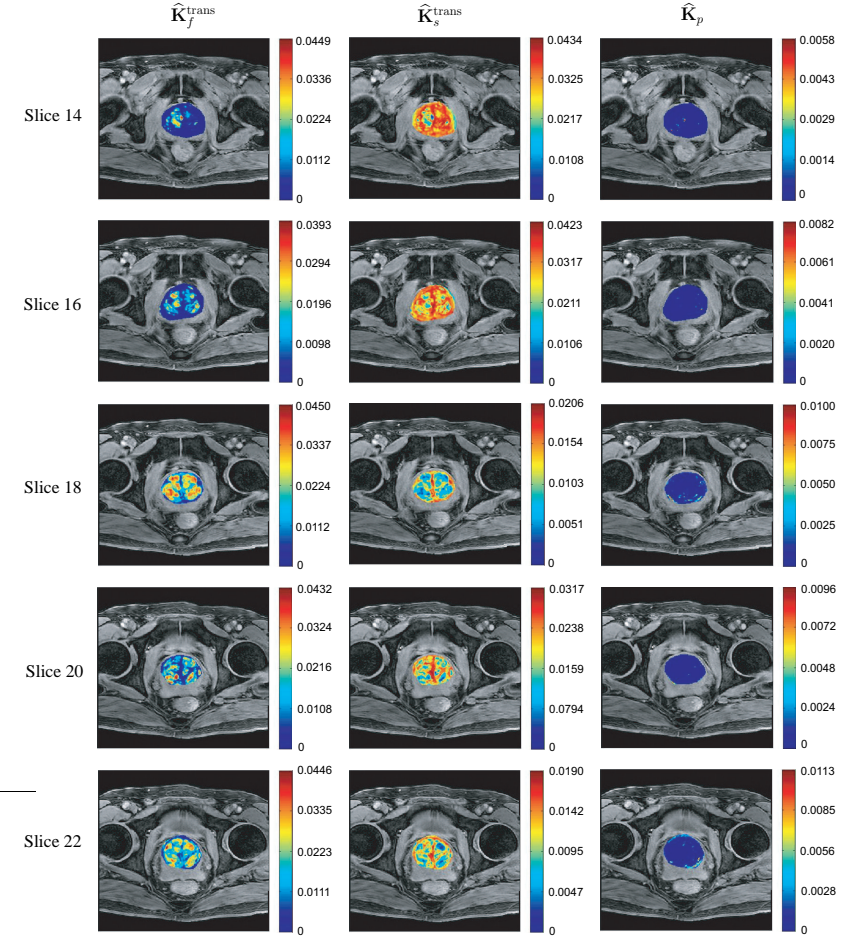

Fig. 7. The KP Maps Estimated by COKE algorithm for Patient B in different slices.

\section{CONCLUSION}

We have presented an effective, convex optimization based BSS algorithm, namely COKE algorithm for PK analysis of prostate cancer using T1 weighted DCE-MRI data. We have first transformed the three-tissue compartmental pharmacokinetic model to a latent variable model so that the PK analysis can be reformulated into a BSS problem. Under the assumption that the tumor is present in the prostate region, the COKE algorithm, first applies normalization to the observed data and successively identifies the pure pixel indices corresponding to the TACs of the fast flow, slow flow, and plasma. The FRCs, which are the most important parameters that determine the accuracy of the estimated KP maps, are then effectively (without local optimality issue and with due considerations for the scaling ambiguities) estimated by solving the original nonconvex FRCs estimation problem, by optimally solving the 


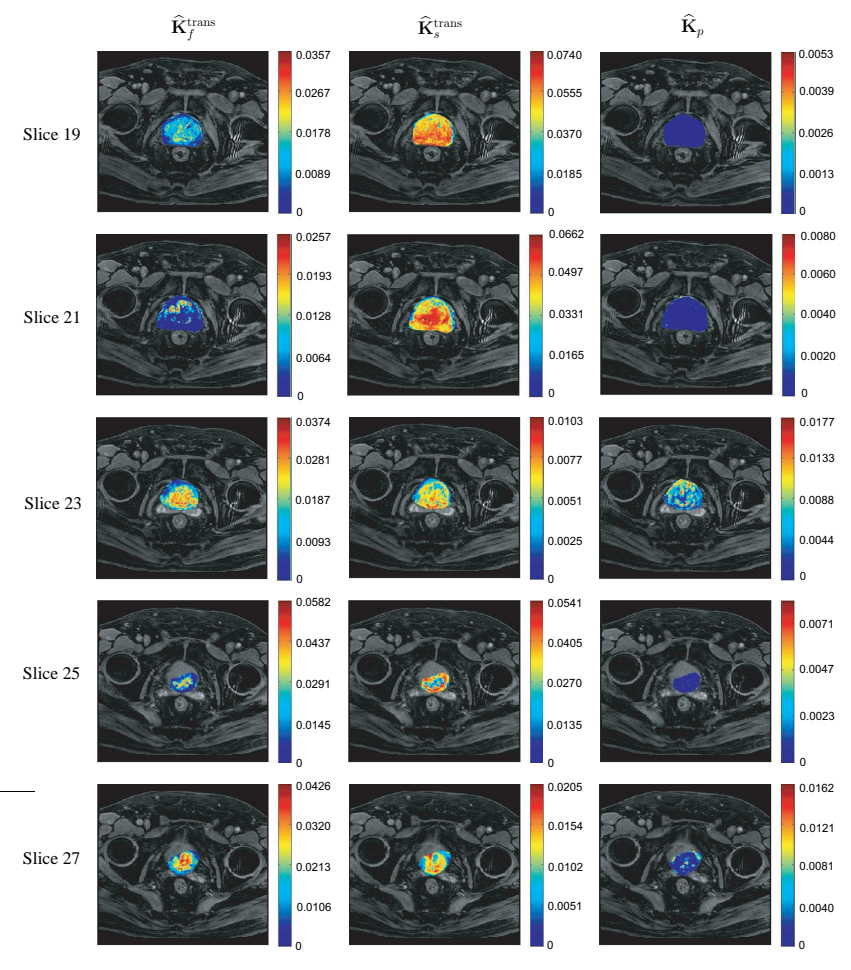

Fig. 8. The KP Maps Estimated by COKE algorithm for Patient $\mathrm{C}$ in different slices.

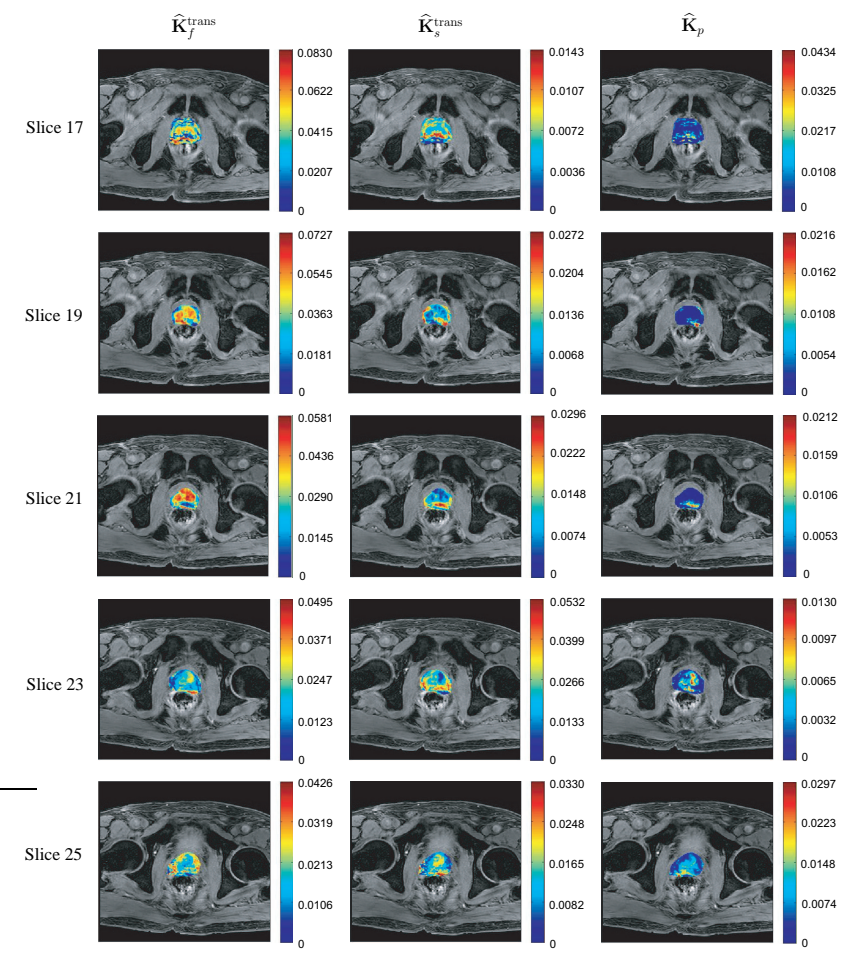

Fig. 9. The KP Maps Estimated by COKE algorithm for Patient D in different slices. corresponding convex optimization problems (32) and (37). Finally, the KP maps are obtained by solving a pixel-wise constrained least-squares optimization (also convex) problem (38), subject to all the possible physical constraints. We have evaluated the efficacy of the COKE algorithm with the synthetic DCE-MRI data and real DCE-MRI data of four patients with prostate cancer. Simulation results have shown that the proposed COKE algorithm performs well for all the scenarios (early-stage, moderate, and advanced tumor). For real data experiments, we have detected the tumor regions together with the estimated TACs and FRCs, which are consistent with the Mackay Memorial Hospital experts team's observations.

Like any other PK analysis algorithm, there is also scope of extension for the proposed COKE algorithm. The proposed COKE algorithm is based on the generalized compartmental model for slice-by-slice PK analysis of DCE-MRI data, thus allowing the inter-slice heterogeneity (i.e., modeling the flux rate constants such that they are allowed to vary between slices, while remaining same within a slice). The most challenging future extension of this work shall be the joint modeling and consideration of both inter-slice and intraslice tumor heterogeneity (wherein the flux rate constants vary within each slice and across slices). The automatic selection of ROI, other modeling based or in situ based measurements for AIF estimation, the reconstruction of 3-D cancer tissue patterns over all the slices based on the observed fast flow KP maps of multiple slices, and pharmacokinetic analysis using the recently developed Magnetic Resonance Fingerprinting (MRF) [38], are also some of the potential future research directions. Finally, formulating and designing an algorithm exclusively for detecting the presence or absence of cancer, and the quantification of the tumor lesions (if identified as cancer) will be of high practical interest.

\section{ACKNOWLEDGEMENT}

This work is partly supported by the National Science Council of Taiwan (R.O.C.) under Grants NSC 102-2221E-007-035-MY2 and MOST 104-2221-E-007-069-MY3, and partly by National Tsing Hua University, Taiwan and Mackay Memorial Hospital, Taiwan, under Grant 100N2742E1. All the DCE-MRI prostate data used in this work are provided by the Mackay Memorial Hospital and appropriate prior approval has been obtained from each Patient. The real data validation and interpretation of the obtained results have been conducted jointly with the Doctors (radiologists) team headed by Dr. FeiShih Yang (one of the co-authors) of the Mackay Memorial Hospital.

\section{REFERENCES}

[1] A. Jemal et al., "Cancer statistics, 2010," CA: A Cancer Journal for Clinicians, vol. 60, no. 5, pp. 277-300, Sep. 2010.

[2] A. L. Potosky et al., "The role of increasing detection in the rising incidence of prostate cancer," The Journal of the American Medical Association, vol. 273, no. 7, pp. 548-552, 1995.

[3] N. B. Deongchamps, A. Singh, and G. P. Haas, "Epidemiology of prostate cancer in Africa: Another step in the understanding of the disease?," Current Problems in Cancer, vol. 31, no. 3, pp. 226-236, June 2007. 
[4] M. A. Khan and A. W. Partin, "Management of patients with an increasing prostate-specific antigen after radical prostatectomy," Current Urology Reports, vol. 3, no. 3, pp. 179-187, Jun. 2004.

[5] L. R. Kavoussi et al., "Complications of laparoscopic pelvic lymph node dissection," Journal of Urology, vol. 149, no. 2, pp. 322-325, Feb. 1993.

[6] C. E. Neal and L. C. Meis, "Correlative imaging with monoclonal antibodies in colorectal, ovarian, and prostate cancer," Seminars in Nuclear Medicine, vol. 24, no. 4, pp. 272-285, Oct. 1994.

[7] K. Lovett et al., "MR imaging characteristics of noncancerous lesions of the prostate," Journal of Magnetic Resonance Imaging, vol. 2, no. 1, pp. 35-39, Jan. 1992

[8] M. L. Schiebler et al., "Prostatic carcinoma and benign prostatic hyperplasia: Correlation of high-resolution MR and histopathologic findings," Radiology, vol. 172, no. 1, pp. 131-137, July 1989.

[9] J. P. B. O'Connor et al., "Dynamic contrast-enhanced MRI in clinical trials of antivascular therapies," Nat. Rev. Clin. Oncol., vol. 9, pp. 167177, Feb. 2012.

[10] C.-A. Cuenod and D. Balvay, "Perfusion and vascular permeability: Basic concepts and measurement in DCE-CT and DCE-MRI," Diagnostic and Interventional Imaging Volume, vol. 94, no. 12, pp. 1187-1204, Dec. 2013.

[11] H. J. Weinmann et al., "Characteristics of GadoliniumDTPA complex: A potential NMR contrast agent," AJR American Journal of Roentgenology, vol. 142, no. 3, pp. 619-624, Mar. 1984.

[12] P. S. Tofts, "Modeling tracer kinetics in dynamic Gd-DTPA MR imaging," Journal of Magnetic Resonance Imaging, vol. 7, no. 1, pp. 91-101, Jan. 1997.

[13] J. O. Barentsz et al., "Fast dynamic Gadolinium-enhanced MR imaging of urinary bladder and prostate cancer," Journal of Magnetic Resonance Imaging, vol. 10, no. 3, pp. 295304, Sep. 1999.

[14] P. L. Choyke, A. J. Dwyer, and M. V. Knopp, "Functional tumor imaging with dynamic contrast-enhanced magnetic resonance imaging," Journal of Magnetic Resonance Imaging, vol. 17, no. 5, pp. 509-520, May 2003

[15] R. Alonzi et al., "Physiological changes within the prostate caused by androgen withdrawal," Clinical Oncology, vol. 19, no. 3, pp. S1-S6, Apr. 2007.

[16] P. S. Tofts et al., "Estimating kinetic parameters from dynamic contrastenhanced T(1)-weighted MRI of a diffusable tracer: Standardized quantities and symbols," Journal of Magnetic Resonance Imaging, vol. 10, no. 3, pp. 223-232, 1999.

[17] Y. Wang et al., "Modeling and reconstruction of mixed functional and molecular patterns," International Journal of Biomedical Imaging, Article ID: 29707, pp. 1-9, 2006.

[18] L. Chen et al., "Tissue-specific compartmental analysis for dynamic contrast-enhanced MR imaging of complex tumors," IEEE Trans. Medical Imaging, vol. 30, no. 12, pp. 2044-2058, Dec. 2011.

[19] Z. J. Wang, "Simultaneous estimation of kinetic parameters and the input function from DCE-MRI data: Theory and simulation," in Proc. IEEE International Symposium on Biomedical Imaging: From Nano to Macro (ISBI), Arlington, VA, USA, April 15-18, 2004, pp. 996-999.

[20] D. Y. Riabkov and E. V. R. Di Bella, "Estimation of kinetic parameters without input functions: Analysis of three methods for multichannel blind identification," IEEE Trans. Biomedical Engineering, vol. 49, no.11, pp. 1318-1327, 2002.

[21] M. D. Plumbley, "Algorithms for non-negative independent component analysis," IEEE Trans. Neural Netw., vol. 14, no. 3, pp. 534-543, May 2003.

[22] D. D. Lee and H. S. Seung, "Learning the parts of objects by nonnegative matrix factorization," Nature, vol. 401, no. 6755, pp. 788-791, Aug. 1999.

[23] A. Ambikapathi et al., "Two effective and computationally efficient purepixel based algorithms for hyperspectral endmember extraction," in Proc. 2011 IEEE ICASSP, Prague, Czech Republic, May 22-27, 2011, pp. $1369-1372$.

[24] J. M. Bioucas-Dias et al., "Hyperspectral unmixing overview: Geometrical, statistical, and sparse regression-based approaches," IEEE J. Sel. Topics in Applied Earth Obs. and Remote Sensing, vol. 5, no. 2, pp. 354-379, June 2012.

[25] A. Ambikapathi et al., "An nBSS algorithm for pharmacokinetic analysis of prostate cancer using DCE-MR images," in Proc. IEEE International Symposium on Biomedical Imaging: From Nano to Macro (ISBI), Barcelona, Spain, May 2-5, 2012, pp. 566-569.
[26] J. F. Sturm, "Using SeDuMi 1.02, a MATLAB toolbox for optimization over symmetric cones," Optimization Methods and Software, vol. 11, no. 12, pp. 625-653, 1999.

[27] M. Grant and S. Boyd. CVX: Matlab software for disciplined convex programming, version 1.21. http://cvxr.com/cvx, Oct. 2010.

[28] P. S. Tofts and A. G. Kermode, "Measurement of the blood-brain barrier permeability and leakage space using dynamic MR imaging 1. Fundamental concepts," Magnetic Resonance in Medicine, vol. 17, no. 2, pp. 357-367, Feb. 1991.

[29] L. Chen et al., "CAM-CM: A signal deconvolution tool for in vivo dynamic contrast-enhanced imaging of complex tissues," Bioinformatics, vol. 27, no. 18, pp. 2607-2609, July 2011.

[30] T.-H. Chan et al., "A simplex volume maximization framework for hyperspectral endmember extraction," IEEE Trans. Geosci. Remote Sens., vol. 49, no. 11, pp. 4177-4193, Nov. 2011.

[31] W.-K. Ma et al., "A signal processing perspective on hyperspectral unmixing," IEEE Signal Process. Mag., vol. 31, no.1, pp. 67-81, Jan. 2014.

[32] S. Boyd and L. Vandenberghe, Convex Optimization. Cambridge, 2004.

[33] T.-H. Chan et al., "A convex analysis framework for blind separation of non-negative sources," IEEE Trans. Signal Processing, vol. 56, no. 10, pp. 5120-5134, Oct. 2008.

[34] Y.-C. Lin et al., "Blind estimation of arterial input function in dynamic contrast-enhanced MRI using purity maximization," Magnetic Resonance in Medicine, vol. 68, no. 5, pp. 1439-1449, Nov. 2012.

[35] A. Ambikapathi et al., "Hyperspectral data geometry based estimation of number of endmembers using p-norm based pure pixel identification," IEEE Trans. Geosci. Remote Sens., vol. 51, no. 5, pp. 2753-2769, May 2013.

[36] C. Yang et al., "Estimating the arterial input function using two reference tissues in dynamic contrast-enhanced MRI studies: Fundamental concepts and simulations," Magnetic Resonance in Medicine, vol. 52, no. 5, pp. 1110-1117, Nov. 2004.

[37] P. A. Humphrey, "Gleason grading and prognostic factors in carcinoma of the prostate," Modern Pathology, vol. 17, pp. 292-306, Feb. 2004.

[38] D. Ma et al., "Magnetic resonance fingerprinting," Nature, vol. 495, no. 7440, pp. 187-192, March 2013.

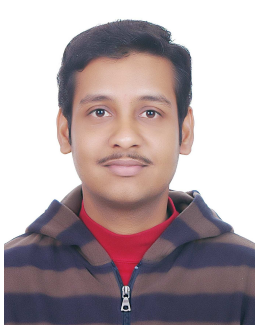

ArulMurugan Ambikapathi (S'02-M'11) received the B.E. degree in electronics and communication engineering from Bharathidasan University, Tiruchirappalli, India, in 2003, the M.E degree in communication systems from Anna University, Chennai, India, in 2005, and the Ph.D. degree from the Institute of Communications Engineering (ICE), National Tsing Hua University (NTHU), Hsinchu, Taiwan, in 2011. He is currently a Senior Algorithm Engineer at Utechzone Co. Ltd., Taipei, Taiwan. He was a Postdoctoral Research Fellow with ICE, NTHU, from Sep. 2011 to Aug. 2014. His research interests are in hyperspectral and biomedical image analysis, convex analysis and optimization for blind source separation, with recent emphasis on automated object identification and computer vision applications.

Dr. Ambikapathi was the recipient of Gold and Silver medals for academic excellence in his B.E and M.E programs, respectively. He was also the recipient of the NTHU Outstanding Student Scholarship award for two consecutive years (2009 and 2010). He was a co-recipient of the IEEE Workshop on Hyperspectral Image and Signal Processing: Evolution in Remote Sensing 2011, Best Paper Award. He was awarded "The Best Ph.D Thesis Award" from IEEE GeoScience and Remote Sensing Society,Taipei Chapter. 


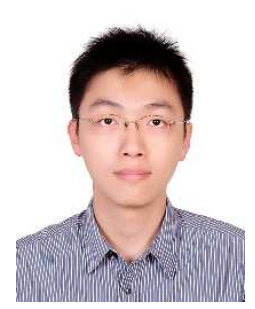

Tsung-Han Chan (S'08-M'09) received his B.S. degree from the Department of Electrical Engineering, Yuan Ze University, Taiwan, in 2004 and his Ph.D. degree from the Institute of Communications Engineering, National Tsing Hua University, Taiwan, in 2009. He is currently working as a Senior Engineer with MediaTek Inc., Hsinchu, Taiwan. He was a corecipient of a WHISPERS 2011 Best Paper Award. He was also recognized as an Outstanding Reviewer for CVPR 2014, and a Best Reviewer of the IEEE TGRS 2014. His research interests are in image processing and numerical optimization, with a recent emphasis on computer vision, machine learning and hyperspectral remote sensing.

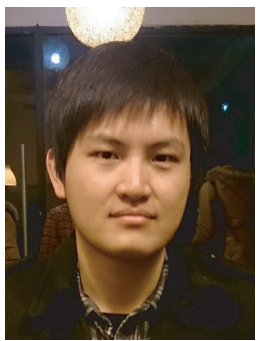

Chia-Hsiang Lin received the B.S. degree in electrical engineering from the National Tsing Hua University, Hsinchu, Taiwan, in 2010, where he is currently working toward the Ph.D. degree in communications engineering. He is currently a visiting Doctoral Graduate Research Assistant with Virginia Polytechnic Institute and State University, Arlington, VA, USA. His research interests are network science, game theory, convex geometry and optimization, and blind source separation.

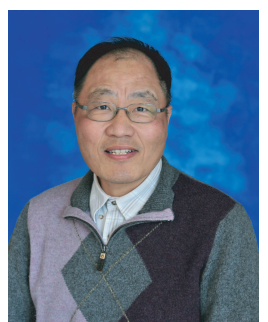

Chong-Yung Chi (S'83-M'83-SM'89) received the Ph.D. degree in Electrical Engineering from the University of Southern California, Los Angeles, California, in 1983. From 1983 to 1988, he was with the Jet Propulsion Laboratory, Pasadena, California. $\mathrm{He}$ has been a Professor with the Department of Electrical Engineering since 1989 and the Institute of Communications Engineering (ICE) since 1999 (also the Chairman of ICE during 2002-2005), National Tsing Hua University, Hsinchu, Taiwan. He has published more than 210 technical papers, including more than 75 journal papers (mostly in IEEE Trans. Signal Processing), 4 book chapters and more than 130 peer-reviewed conference papers, as well as a graduate-level textbook, Blind Equalization and System Identification, Springer-Verlag, 2006. His current research interests include signal processing for wireless communications, convex analysis and optimization for blind source separation, biomedical and hyperspectral image analysis.

Dr. Chi is a senior member of IEEE. He has been a Technical Program Committee member for many IEEE sponsored and co-sponsored workshops, symposiums and conferences on signal processing and wireless communications, including Co-organizer and General Co-chairman of 2001 IEEE Workshop on Signal Processing Advances in Wireless Communications (SPAWC), and Co-Chair of Signal Processing for Communications (SPC) Symposium, ChinaCOM 2008 and Lead Co-Chair of SPC Symposium, ChinaCOM 2009. He was an Associate Editor (AE) of IEEE Trans. Signal Processing (5/2001 4/2006), IEEE Trans. Circuits and Systems II (1/200612/2007), IEEE Trans. Circuits and Systems I (1/2008-12/2009), AE of IEEE Signal Processing Letters (6/2006 5/2010), and a member of Editorial Board of Signal Processing (6/2005 5/2008), and an editor (7/2003 12/2005) as well as a Guest Editor (2006) of EURASIP Journal on Applied Signal Processing. He was a member of Signal Processing Theory and Methods Technical Committee (SPTM-TC) (2005-2010), IEEE Signal Processing Society. Currently, he is a member of Signal Processing for Communications and Networking Technical Committee (SPCOM-TC) and a member of Sensor Array and Multichannel Technical Committee (SAM-TC), IEEE Signal Processing Society, and an AE of IEEE Trans. Signal Processing.

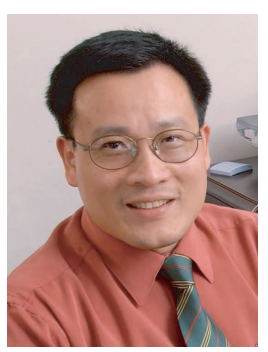

Yue Wang received his B.S. and M.S. degrees in electrical and computer engineering from Shanghai Jiao Tong University in 1984 and 1987 respectively. $\mathrm{He}$ received his Ph.D. degree in electrical engineering from University of Maryland Graduate School in 1995. He is currently the endowed Grant A. Dove Professor of electrical and computer engineering at Virginia Polytechnic Institute and State University. His research interests focus on machine learning, pattern recognition, signal and image processing, with applications to biomedical sciences.

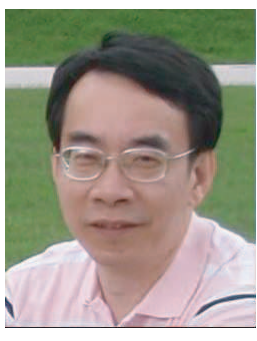

Fei-Shih Yang was born in Taichung, Taiwan. He received and completed his medical degree in 1983 from China medical university, Taichung, Taiwan. He was trained in radiology in Mackay Memorial Hospital (MMH) at Taipei, Taiwan from 1983 to 1987. From 1987 onward, he took positions as an Attending Staff Radiologist, Department of Radiology, MMH (Taipei). Since 2006 he was as a department chief of radiology, MMH (Taipei). He was also an instructor in Yuanpei University from 1998 to 2012 and Mackay Memorial medical college since 2012

to present

Dr Yang is an expert in diagnostic abdominal imaging and abdominal interventional radiology in MMH more than 25 years. He has published more than 40 peer-reviewed research articles in English or Chinese. 